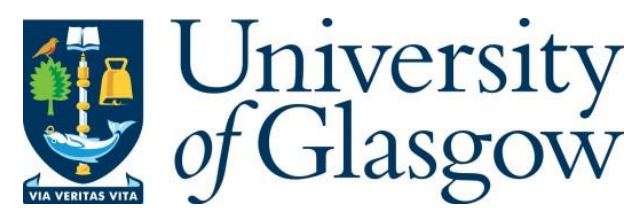

Qu, H., Liu, G., Zhang, L., Wen, S. and Imran, M. A. (2020) Low-complexity symbol detection and interference cancellation for OTFS system. IEEE Transactions on Communications, (doi: 10.1109/TCOMM.2020.3043007).

There may be differences between this version and the published version. You are advised to consult the publisher's version if you wish to cite from it.

http://eprints.gla.ac.uk/226632/

Deposited on: 9 December 2020

Enlighten - Research publications by members of the University of Glasgow http://eprints.gla.ac.uk 


\title{
Low-Complexity Symbol Detection and Interference Cancellation for OTFS System
}

\author{
Huiyang Qu, Guanghui Liu, Senior Member, IEEE, Lei Zhang, Senior Member, IEEE, \\ Shan Wen, Student Member, IEEE, and Muhammad Ali Imran, Senior Member, IEEE
}

\begin{abstract}
Orthogonal time frequency space (OTFS) is a twodimensional modulation scheme realized in the delay-Doppler domain, which targets the robust wireless transmissions in highmobility environments. In such scenarios, OTFS signal suffers from multipath channel with continuous Doppler spread, which results in significant inter-symbol interference and inter-Doppler interference (IDI). In this paper, we analyze the interference generation mechanism, and compare statistical distributions of the IDI in two typical cases, i.e., limited-Doppler-shift channel and continuous-Doppler-spread channel (CoDSC). Focusing on the OTFS signal transmission over the CoDSC, our study firstly indicates that the widespread IDI incurs a computational burden for the element-wise detector like the message passing in the state-of-the-art works. Addressing this challenge, we propose a block-wise OTFS receiver by exploiting the structure and characteristics of the OTFS transmission matrix. In the receiver, we deliberately design an iteration strategy among the least squares minimum residual based channel equalizer, reliability-based symbol detector and interference eliminator, which can realize fast convergence by leveraging the sparsity of channel matrix. The simulations demonstrate that, in the CoDSC, the proposed scheme achieves much less detection error, and meanwhile reduces the computational complexity by an order of magnitude, compared with the state-of-the-art OTFS receivers.
\end{abstract}

Index Terms-Orthogonal time frequency space (OTFS), least squares minimum residual (LSMR), channel equalization, high mobility, continuous-Doppler-spread channel (CoDSC).

\section{INTRODUCTION}

Reliable data transmission in high-mobility environments, such as high-speed railways [1], [2], vehicular to everything network (V2X) [3]-[5], and unmanned aerial vehicles [6], [7], has been recognized as an important requirement in future wireless communications. One of the main challenges in these scenarios is estimation and equalization of highly doubly-dispersive radio channel in the time-frequency (TF) domain. The challenge poses a bottleneck to the overall system performance when the traditionally one-dimensional time or frequency modulation scheme is adopted. Recently, a two-dimensional (2-D) modulation technique, namely orthogonal time frequency space (OTFS), has been proposed

This work was supported in part by the National Natural Science Foundation of China under Grant 62071097, and in part by the 111 Project under Grant B17008. (Corresponding author: Guanghui Liu).

Huiyang Qu, Guanghui Liu and Shan Wen are with the School of Information and Communication Engineering, University of Electronic Science and Technology of China, Chengdu 611731, China (e-mail: guanghuiliu@uestc.edu.cn; \{hyqu, shanwen\}@std.uestc.edu.cn).

Lei Zhang and Muhammad Ali Imran are with the James Watt School of Engineering, University of Glasgow, Glasgow, G12 8QQ, U.K. (e-mail: \{Lei.Zhang, Muhammad.Imran\}@glasgow.ac.uk). to improve the wireless transmission performance in highmobility scenarios [8]. Unlike the TF-domain scheme adopted in orthogonal frequency division multiplexing (OFDM) [9][11], OTFS constructs a delay-Doppler (DD) transmission scheme by multiplexing the information symbols in a DD plane. In high mobility, the sparsity and lower variability of the channel in the DD domain simplify channel estimation and signal detection [12]. Benefitting from that, OTFS can reap the full channel diversity more efficiently than OFDM, yielding superior transmission performance [13].

The (inverse) symplectic finite Fourier transform (ISFFT/SFFT) builds a bridge between the DD (TF) domain and TF (DD) domain, which enables the OTFS to be implemented by a simple pre-processing step over the TF-domain multi-carrier scheme such as OFDM [14], [15]. As a Fourier duality of TF domain, the DD-domain transmission can be expressed by a 2-D convolution between DD channel and OTFS symbols [16]. For a multipath channel with nonzero Doppler spread, this 2-D convolution makes each OTFS symbol diffuse along both the delay and Doppler dimensions. As a result, the diffusion causes the inter-symbol interference (ISI) and inter-Doppler interference (IDI), respectively distributing in the delay and Doppler dimensions [17]. The higher the channel delay and Doppler spreads are, the severer ISI and IDI are in OTFS systems. Therefore, in high-mobility environments, the interference cancellation and signal detection are indispensable for realizing robust OTFS data transmission.

Several OTFS signal detectors have been proposed in the literature, including non-linear schemes [18]-[22] and linear schemes [23]-[25]. These methods are based on an assumption that the OTFS signal undergoes the limited-Doppler-shift channel (LDSC), where the channel spectrum appears as a few impulses in the Doppler dimension [18]. Unfortunately, the assumption is only suitable for the sparse-scattering environments, for instance, high-speed train running in a clear and open signal-transmission space [26]. In contrast, in some mobile transmissions, such as vehicular communications with large number of surroundings [27], there are abundant channel scatters. The channel spectrum always occupies a continuous range in the Doppler frequency. To our best knowledge, in OTFS transmission, there is no research focusing on the continuous-Doppler-spread channel (CoDSC). The traditional demodulation algorithms, developed for the LSDC, cannot directly used in the CoDSC for achieving the desired demodulation performance and/or implementation complexity. Specifically, although the widely-used linear schemes [23]- 
[25] are easy to be implemented, they can not achieve the ideal detection performance, in contrast to the state-of-the-art message passing (MP) based non-linear equalization schemes [18]-[20]. In the CoDSC, however, the MP detector involves an unacceptable computation burden, since its complexity highly relies on the number of channel scatters.

Recognizing the challenges for the OTFS demodulation in the CoDSC, this paper proposes a low-complexity OTFS receiver, which is constructed by the least squares minimum residual (LSMR) based channel equalizer, dynamic symbol detector, and joint ISI and IDI eliminator, to iteratively perform data detection and interference cancellation. With only a few iterations, the transmitted quadrature amplitude modulation (QAM) symbols can be ideally retrieved. The proposed algorithm, with linear complexity, can be more efficiently implemented, compared with the factor graph based interference cancellation in the MP [18]-[20]. Specifically, our contributions are summarized as follows.

1) It is the first time to model the OTFS signal transmission over CoDSC. We mathematically derive the generation mechanism of the IDI, and further analyze the statical properties of the interference in the CoDSC and LDSC. The analysis provides useful insights into designing channel equalization in the scatter-abundant scenarios.

2) An efficient block-wise receiver architecture involving a joint ISI and IDI eliminator is proposed to enhance the OTFS performance in CoDSC. Based on the observation, that the OTFS channel matrix is large size, sparse and ill-conditioned, we develop an LSMR based channel equalizer, which leverages a reliabilitybased dynamic detector to retrieve the transmitted QAM symbols, instead of adopting the traditional hard or soft decision of the symbol estimates. The proposed iteration scheme between the symbol detection and interference cancellation converges quickly by leveraging the sparsity of the OTFS transmission matrix, which enables the OTFS receiver to work efficiently in the CoDSC.

3) We build the OTFS simulation platform and investigate demodulation performance of the proposed OTFS receiver in the CoDSC. The simulation results demonstrate the proposed receiver is capable of achieving the superior demodulation performance to the existing OTFS equalizers. For example, in the 64-QAM OTFS system, the proposed receiver achieves at least $5 \mathrm{~dB}$ signal-to-noise ratio (SNR) gain, in the CoDSC with a velocity of $540 \mathrm{~km} / \mathrm{h}$, compared with the MP based receiver [18]. Meanwhile, thanks to the much fewer iteration times, the required detection complexity is reduced by an order of magnitude.

The remainder of this paper is organized as follows. The OTFS signaling and the DD channel are formulated in Sec. II. In Sec. III, the IDI generation mechanisms are presented. The OTFS receiver is developed in Sec. IV, followed by simulation results in Sec. V. Finally, this paper is concluded in Sec. VI.

Notations: In this paper, we use upper (lower) boldface letters to denote matrices (column vectors). $Y(l, v)$ denotes the element in the $l$-th row and $v$-th column of matrix $\mathbf{Y}$. The $l$-th element in vector $\mathbf{y}$ is denoted as $\mathbf{y}(l)$. Vectors $\mathbf{X}(n,:)$ and $\mathbf{X}(:, n)$ denote the $n$-th row and $n$-th column of $\mathbf{X}$, respectively. We use $(\cdot)^{-1},(\cdot)^{T}$ and $(\cdot)^{H}$ to represent inverse, transpose and conjugate transpose, respectively. The operators $\operatorname{vec}(\cdot),\|\cdot\|$, Cond $(\cdot)$ and $\mathbb{E}\{\cdot\}$ are denoted as the vectorizing of a matrix, Frobenius norm, the condition number of a matrix, and expectation operation, respectively. $\delta(\cdot)$ represent unit impulse function. $\mathbb{C}$ stands for the set of complex numbers. $\mathbf{I}_{M}$ denotes the $M \times M$ identity matrix. The zeroth order Bessel function of the first kind is denoted as $J_{0}(\cdot) . \mathbf{F}_{M}$ and $\mathbf{F}_{N}$ denote the fast Fourier transform (FFT) matrices, which are composed of $F_{M}(m, k)=\frac{1}{\sqrt{M}} \exp (-j 2 \pi m k / M)$ and $F_{N}(n, k)=\frac{1}{\sqrt{N}} \exp (-j 2 \pi n k / N)$, respectively.

\section{OTFS TRANSMISSION ARCHITECTURE}

The discrete-time OTFS transmission model is depicted in Fig. 1. The DD-domain data, composed of QAM symbols, are arranged into a data matrix $\mathbf{X} \in \mathbb{C}^{M \times N}$, where $M$ and $N$ are numbers of resource grids along the delay and Doppler dimensions, respectively. The ISFFT converts $\mathbf{X}$ from the DD domain into TF domain, i.e, $\mathbf{D}=\mathbf{F}_{M} \mathbf{X F}_{N}^{H}$. Subsequently, the time-domain data block is generated by $\mathbf{S}=\mathbf{F}_{M}^{H} \mathbf{D}$. Each column of $\mathbf{S}$, i.e., $\mathbf{S}(:, n)$, is treated as a time-domain OFDM symbol. The CP with length $M_{c p}$ is inserted at the beginning of each symbol to avoid ISI. The discrete-time OFDM sequences go through the doubly selective channel, of which the impulse response is defined as $h\left[n, l^{\prime}\right], l^{\prime} \in[0, L-1]$, where the maximum channel delay spread $L \leq M_{c p}$. Assuming the channel observes typical wide-sense stationary uncorrelated scattering model, we have

$$
\mathbb{E}\left\{h\left[n, l^{\prime}\right] h^{*}\left[m, l^{\prime \prime}\right]\right\}=\sigma_{l^{\prime}}^{2} r(n-m) \delta\left(l^{\prime}-l^{\prime \prime}\right) .
$$

In (1), $\sigma_{l^{\prime}}^{2}$ denotes the power of the $l^{\prime}$-th propagation path; $r(n-m)$ denotes the normalized tap autocorrelation, where $r(0)=1$.

At the receiver side, after removing $\mathrm{CP}$, the received timedomain data block $\mathbf{R} \in \mathbb{C}^{M \times N}$ is converted into the TF domain by means of FFT in OFDM demodulator, which is expressed as $\tilde{\mathbf{D}}=\mathbf{F}_{M} \mathbf{R}$. Then, the SFFT converts the TFdomain data block $\tilde{\mathbf{D}}$ back into the DD domain, according to $\mathbf{Y}=\mathbf{F}_{M}^{H} \tilde{\mathbf{D}} \mathbf{F}_{N}$.

Proposition 1: We denote the $(l, v)$-th element of $\mathbf{Y}$ as $Y(l, v), l \in[0, M-1], v \in[0, N-1]$. It follows

$Y(l, v)=\sum_{v^{\prime}=0}^{N-1} \sum_{l^{\prime}=0}^{M-1} \Omega\left\{l,\left(l-l^{\prime}\right)_{M}, v-v^{\prime}\right\} X\left(l^{\prime}, v^{\prime}\right)+W(l, v)$,

where $\Omega\left\{l, l^{\prime}, v^{\prime}\right\}$ is the DD-domain channel response

$\Omega\left\{l, l^{\prime}, v^{\prime}\right\}=\frac{1}{N} \sum_{i=0}^{N-1} e^{\frac{-j 2 \pi i v^{\prime}}{N}} h\left[i\left(M+M_{c p}\right)+M_{c p}+l, l^{\prime}\right]$,

$W(l, v)$ represents the additive white Gaussian noise with zero mean and variance $\sigma^{2} ; X\left(l^{\prime}, v^{\prime}\right), l^{\prime} \in[0, L-1], v^{\prime} \in$ 


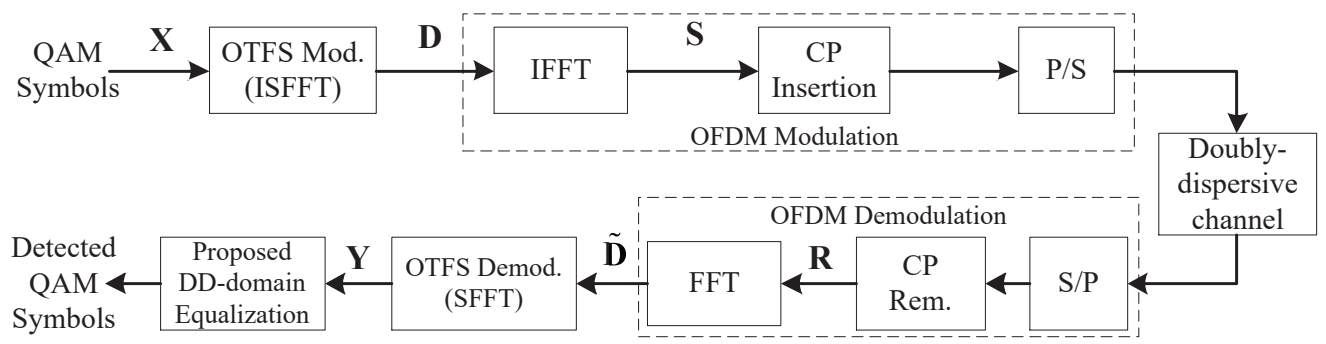

Fig. 1. The baseband transmission model of OTFS system.

$[0, N-1]$ denotes the $\left(l^{\prime}, v^{\prime}\right)$-th entry of $\mathbf{X}$. Proposition 1 is proofed in Appendix A.

We rewrite (2) as

$$
Y(l, v)=\Omega\{l, 0,0\} X(l, v)+I(l, v)+W(l, v),
$$

where the fisrt term in the right-hand side (RHS) of (4) denotes the desired signal component, and the second term $I(l, v)$ represents the interference, which is expressed in (5).

It can be seen from (5) that $I(l, v)$ contains the ISI and IDI components. The ISI is caused by the spread of OTFS signal along the delay dimension. If $L=0$, the time-domain CIR is rudeced to $h\left[n, l^{\prime}\right]=h[n] \delta\left(l^{\prime}\right)$. According to (3) and (5), only the second term in the RHS of (5) exists in this case. In the next section, we will analyze the characteristics of the IDI, which can provide some insights into the equalization of the OTFS signal.

\section{ANALYSIS OF INTER-DOPPLER INTERFERENCE}

The generation mechanism and distribution of the IDI are analyzed in this section, by means of investigating its second-order statistics, i.e., interference power. Comparing the IDI in two typical channels, i.e., LDSC and CoDSC, we find the continuous channel Doppler spectrum incurs a widespread IDI in OTFS system, posing a challenge to channel equalization.
From (1) and (3), the power of DD-domain channel gains, i.e., $\mathbb{E}\left\{\left|\Omega\left\{l,\left(l-l^{\prime}\right)_{M}, v-v^{\prime}\right\}\right|^{2}\right\}$ is derived as (6). Equ. (6) can be further rewritten as (7), where $q=i-s$, autocorrelation function $r_{t}(q)$ follows

$$
r_{t}(q)=r_{t}(i-s)=r\left((i-s)\left(M+M_{c p}\right)\right),
$$

and

$$
\omega_{q}=\left\{\begin{array}{cc}
N-|q|, & -N<q<N \\
0, & \text { else }
\end{array} .\right.
$$

For simplicity, we suppose $\mathbb{E}\left\{\left|X\left(l^{\prime}, v^{\prime}\right)\right|^{2}\right\}=1$.

IDI-generating mechanism: The second and third terms in the RHS of (5) contain IDI. It generates from the diffusion of OTFS symbols along the Doppler dimension. Based on the channel power in (7) and IDI components in (5), the IDI power $\Xi_{N}^{\text {IDI }}\left(v-v^{\prime}\right)$ is expressed as

$$
\Xi_{N}^{\mathrm{IDI}}\left(v-v^{\prime}\right)=\frac{1}{N^{2}} \sum_{q} \omega_{q} r_{t}(q) e^{-j 2 \pi\left(v-v^{\prime}\right) q / N} .
$$

The term in the RHS of (28) can be rewritten in a form of convolution by applying the Fourier transform to $\omega_{q}$ and $r_{t}(q)$. Thus, we have the following proposition.

Proposition 2: The IDI power is formulated as

$$
\Xi_{N}^{\mathrm{IDI}}\left(v-v^{\prime}\right)=\left.(W(\phi) * R(\phi))\right|_{\phi=v-v^{\prime}},
$$

$$
\begin{aligned}
& I(l, v)=\underbrace{\sum_{l^{\prime}=0, l^{\prime} \neq l}^{M-1} \Omega\left\{l,\left(l-l^{\prime}\right)_{M}, 0\right\} X\left(l^{\prime}, v\right)}_{\text {ISI only }}+\underbrace{\sum_{v^{\prime}=0, v^{\prime} \neq v}^{M-1} \Omega\left\{l, 0, v-v^{\prime}\right\} X\left(l, v^{\prime}\right)}_{\text {IDI only }} \\
& \mathbb{E}\left\{\left|\Omega\left\{l,\left(l-l^{\prime}\right)_{M}, v-v^{\prime}\right\}\right|^{2}\right\}=\sigma_{\left(l-l^{\prime}\right)_{M}}^{2} \frac{1}{N^{2}} \sum_{i=0}^{N-1} \sum_{s=0}^{N-1} e^{\frac{-j 2 \pi(i-s)\left(v-v^{\prime}\right)}{N}} r\left((i-s)\left(M+M_{c p}\right)\right) . \\
& =\underbrace{\sigma_{\left(l-l^{\prime}\right)_{M}}^{2}}_{\text {Delay-domain Power }} \underbrace{\frac{1}{N^{2}} \sum_{q} \omega_{q} r_{t}(q) e^{-j 2 \pi\left(v-v^{\prime}\right) q / N}}_{\text {Doppler-domain Power }} .
\end{aligned}
$$


where $R(\phi)$ is the channel Doppler spectrum

$$
R(\phi)=\sum_{q} r_{t}(q) e^{-j 2 \pi \phi q / N},
$$

and $W(\phi)$ denotes the discrete-time Fourier transform of $\omega_{q} / N^{2}$, i.e.,

$$
W(\phi)=\frac{1}{N^{2}} \sum_{q} \omega_{q} e^{-j 2 \pi \phi q / N}=(\operatorname{Diric}(2 \pi \phi / N))^{2} .
$$

In (13),

$$
\operatorname{Diric}(x)=\frac{\sin (N x / 2)}{N \sin (x / 2)},
$$

which is commonly known as a Dirichlet sinc [9].

From 11 , the IDI power $\Xi_{N}^{\text {IDI }}(\phi)$ is the convolution between the channel Doppler spectrum $R(\phi)$ and function $W(\phi)$. The distribution of $\Xi_{N}^{\text {IDI }}\left(v-v^{\prime}\right)$ highly depends on the channel Doppler spectrum $R(\phi)$. In some high-mobility environments, for instance, the high-speed trains running in the clear and open signal-transmission spaces, the channel scattering components are very limited, and so are the channel Doppler shifts. It means $R(\phi)$ always consists of a small number of frequency elements. However, in some other highmobility scenarios, such as the vehicles moving in the urban areas with lots of surroundings, the channel scattering components are infinite. It means $R(\phi)$ may occupy a continuous range of the Doppler frequency. In this paper, to facilitate the study of IDI, we analyze and compare the IDI distribution in two typical cases, namely LDSC and CoDSC. Each of them shows the exclusive characteristics of $R(\phi)$, thus exposing different IDI power $\Xi_{N}^{\text {IDI }}\left(v-v^{\prime}\right)$.

Case I (LDSC): We give a general CIR expression in the LDSC, and further derive the channel Doppler spectrum $R(\phi)$ to help us analyze the IDI power $\Xi_{N}^{\mathrm{IDI}}\left(v-v^{\prime}\right)$. In the LSDC, the CIR $h\left[n, l^{\prime}\right]$ can be modeled as

$h\left[n, l^{\prime}\right]=\sum_{k=0}^{K-1} \alpha_{l^{\prime}, k} e^{j\left(2 \pi f_{l^{\prime}, k} n T_{s}+\theta_{l^{\prime}, k}\right)} \triangleq \sum_{k=0}^{K-1} \tilde{\alpha}_{l^{\prime}, k} e^{j 2 \pi f_{l^{\prime}, k} n T_{s}}$

where $\tilde{\alpha}_{l^{\prime}, k}=\alpha_{l^{\prime}, k} e^{j \theta_{l^{\prime}, k}}, T_{s}$ is the system sampling period and $K$ denotes the number of Doppler shifts of the transmission path. Parameters $\theta_{l^{\prime}, k}, \alpha_{l^{\prime}, k}$ and $f_{l^{\prime}, k}$ represent the $k$-th initial phase, complex gain and Doppler shift associated with the $l^{\prime}$-th path.

Combining (15) with (1), the autocorrelation function $r_{t}(q)$ in (5) can be expressed as

$$
r_{t}(q)=\sum_{k=0}^{K-1} e^{j 2 \pi f_{l^{\prime}, k} T_{s}\left(M+M_{c p}\right) q} \triangleq \sum_{k=0}^{K-1} e^{j 2 \pi \bar{f}_{l^{\prime}, k} q / N} .
$$

In 16, $\bar{f}_{l^{\prime}, k}=N\left(M+M_{c p}\right) T_{s} f_{l^{\prime}, k}$ denotes the sampled Doppler shift, decomposed as

$$
\bar{f}_{l^{\prime}, k}=\bar{f}_{l^{\prime}, k}^{\mathrm{Int}}+\bar{f}_{l^{\prime}, k}^{\mathrm{Fra}},
$$

where

$$
\bar{f}_{l^{\prime}, k}^{\mathrm{Int}}=\left\lfloor\bar{f}_{l^{\prime}, k}\right\rfloor,\left|\bar{f}_{l^{\prime}, k}^{\mathrm{Int}}\right| \leq N / 2
$$

stands for the index of integer Doppler taps, and $\bar{f}_{l^{\prime}, k}^{\text {Fra }}$ is the fractional part of $\bar{f}_{l^{\prime}, k}$, called as the index of fractional

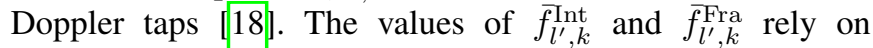
the sampling resolution $N\left(M+M_{c p}\right) T_{s}$ in the Dopplerfrequency direction. By using (16) and (12), the Doppler spectrum is expressed as

$$
R(\phi)=\sum_{k=0}^{K-1} \delta\left(\phi-\bar{f}_{l^{\prime}, k}\right) .
$$

Substituting (13) and (19) into (11), the IDI power $\Xi_{N}^{\text {IDI }}\left(v-v^{\prime}\right)$ in the LDSC is calculated as

$$
\Xi_{N}^{\mathrm{IDI}}\left(v-v^{\prime}\right)=\sum_{k=0}^{K-1}\left[\operatorname{Diric}\left(2 \pi\left(v-v^{\prime}-\bar{f}_{l^{\prime}, k}\right) / N\right)\right]^{2} .
$$

With 17, 20, can be rewritten as

$\Xi_{N}^{\mathrm{IDI}}\left(v-v^{\prime}\right)=\sum_{k=0}^{K-1}\left[\operatorname{Diric}\left(2 \pi\left(v-v^{\prime}-\bar{f}_{l^{\prime}, k}^{\mathrm{Int}}-\bar{f}_{l^{\prime}, k}^{\mathrm{Fra}}\right) / N\right)\right]^{2}$.

In 21, $\Xi_{N}^{\text {IDI }}\left(v-v^{\prime}\right)$ achieves the maximum value when $v-v^{\prime}-\vec{f}_{l^{\prime}, k}^{\mathrm{nt}}=0$ or 1 , and quickly drops to zero as $\left|v-v^{\prime}\right|$ increases. It can be seen from (21) that the IDI in LDSC distributes in a form of Dirichlet sinc and the IDI power depends on the value of fractional Doppler $f_{l^{\prime}, k}^{\mathrm{Fra}}$. In particular, if $\overline{f^{\prime}, k}=0$, we have

$$
\Xi_{N}^{\text {IDI }}\left(v-v^{\prime}\right)=\left\{\begin{array}{rc}
1 / N^{2}, & v-v^{\prime}=\bar{f}_{l^{\prime}, k}^{\text {Int }}, \\
0, & \text { others }
\end{array}\right.
$$

indicating the IDI is not incurred. When $\bar{f}_{l^{\prime}, k}^{\text {Fra }} \neq 0$, the transmitted symbols will diffuse along the entire Doppler dimension, thus involving the IDI.

Case II (CoDSC): As a typical case of the CoDSC, the classical Jakes' channel model [28] is selected to help us analyze the IDI. For $l^{\prime}$-th transmission path, $h\left[n, l^{\prime}\right]$ follows Rayleigh distribution in time, of which the autocorrelation function $r_{t}(q)$ in $(5)$ is

$$
r_{t}(q)=J_{0}\left(2 \pi f_{d} T_{s}\left(M+M_{c p}\right) q\right),
$$

where $f_{d}$ denotes the maximum Doppler frequency, which is normalized to the symbol rate as

$$
\tilde{f}_{d}=f_{d} T_{s}\left(M+M_{c p}\right) .
$$

$$
\begin{aligned}
\Xi_{N}^{\mathrm{IDI}}\left(v-v^{\prime}\right) & =\left.(W(\phi) * R(\phi))\right|_{\phi=v-v^{\prime}} \\
& =\left.\left((\operatorname{Diric}(2 \pi \phi / N))^{2} * \frac{1}{2 \pi \sqrt{\tilde{f}_{d}^{2}-(\phi / N)^{2}}}\right)\right|_{\phi=v-v^{\prime}}
\end{aligned}
$$


The channel Doppler spectrum $R(\phi)$ is computed as

$$
R(\phi)=\left\{\begin{array}{cc}
\frac{1}{2 \pi \sqrt{\tilde{f}_{d}^{2}-(\phi / N)^{2}}},|\phi|<N \tilde{f}_{d} \\
0, & |\phi|>N \tilde{f}_{d}
\end{array},\right.
$$

which is a classical U-shaped (bell-shaped) spectrum extended in the Doppler dimension. Based on (25) and (13), the IDI power $\Xi_{N_{\tilde{N}}}^{\mathrm{IDI}}\left(v-v^{\prime}\right)$ is derived from in (26) and (27).

When $\tilde{f}_{d}=0, R(\phi)$ turns into a single impulse at $\phi=0$, and

$$
\Xi_{N}^{\mathrm{IDI}}\left(v-v^{\prime}\right)=\left[\operatorname{Diric}\left(2 \pi\left(v-v^{\prime}\right) / N\right)\right]^{2} .
$$

No IDI incurs since $\Xi_{N}^{\text {IDI }}\left(v-v^{\prime}\right)=0$ for any integer $v \neq v^{\prime}$. Once $\tilde{f}_{d} \neq 0, R(\phi)$ occupies a continuous interval $-N \tilde{f}_{d}<$ $\phi<N \tilde{f}_{d}$, leading to a spread of $\Xi_{N}^{\text {IDI }}\left(v-v^{\prime}\right)$ in the Doppler dimension. As a result, the IDI will be involved.

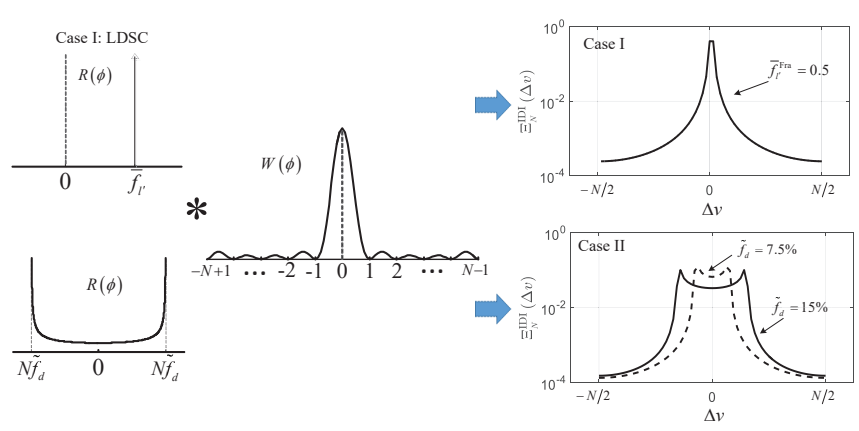

Fig. 2. Illustration of the IDI-generating mechanism (single-Doppler-shift channel (SDSC) and Jakes' channel).

Fig. 22 demonstrates the IDI-generating mechanism in the single-Doppler-shift channel (SDSC) and Jakes' channe 1 respectively, where the Doppler spectrum $R(\phi)$ is convolved by $W(\phi)$. For the SDSC, we consider the integer Doppler $f_{l^{\prime}}^{\text {Int }}=0$ and fractional Doppler $f_{l^{\prime}}^{\text {Fra }}=0.5$ in 17, which results in the most significant IDI power in (21) for all values of $\overline{f_{l^{\prime}}}$ Fra . The ratio of IDI bandwidth to the entire Doppler spectrum, for $\Xi_{N}^{\text {IDI }}(\Delta v) \geq 10^{-2}, \Delta v=v-v^{\prime}$, is about $5 \%$. Hence, the state-of-the-art OTFS receivers that utilizing the MP detection schemes [18]-[20], can model the OTFS transmission as a sparsely-connected factor graph, by ignoring the IDI components for $\Xi_{N}^{\text {IDI }}(\Delta v) \leq 10^{-2}$. In this way, the MP can achieve a satisfactory interference cancellation performance with a relatively low implementation cost thanks to the narrow IDI band. In the Jakes' channel, we choose

\footnotetext{
${ }^{1}$ Here, we take the SDSC and Jakes' channel as examples to illustrate the IDI-generating mechanism in the LDSC and CoDSC cases, respcetively. The SDSC is commonly considered in the existing works, e.g., [15], [18]-[20], [24], 25].
}

$\tilde{f}_{d}$ in 24 as $7.5 \%$ and $15 \%$. It can be seen that the IDI bandwidth for $\Xi_{N}^{\text {IDI }}(\Delta v) \geq 10^{-2}$, are much wider than that in the SDSC. In the high-speed environment, $\tilde{f}_{d}=15 \%$, corresponding to a velocity of $540 \mathrm{~km} / \mathrm{h}$, the ratio becomes more than $30 \%$. Then, in this case, the MP detector causes unacceptable computation burden.

Remark 1: In SDSC, the generation of IDI is not directly related to the UE's velocity but depends on the Dopplerdomain sampling resolution $T_{s}\left(M+M_{c p}\right) N$. In other word$\mathrm{s}$, the distribution of IDI relies on the value of fractional Doppler $\bar{f}_{l^{\prime}}^{\mathrm{Fra}}$. Hence, in practice, $N$ needs to be sufficiently large to achieve an ideal resolution in the Doppler domain, which ensures $\bar{f}_{l^{\prime}}^{\text {Fra }}$ approach to be zero, such that the fractional Doppler incurred IDI can be ignored [29]. In contrast, in the Jakes' channel, IDI is introduced due to the mobility (nonzero Doppler spread). Once the UE is not static, the nulls of $(W(\phi) * R(\phi))$ no longer fall on the Doppler grids, thus introducing IDI. Compared with the single Doppler spectrum, the continuous Doppler spectrum within the interval $-N \tilde{f}_{d}<\phi<N \tilde{f}_{d}$ involves a much wider IDI bandwidth.

\section{Symbol Detection And Interference CANCEllation}

The analysis of the interference reveals that the IDI cancellation in CoDSC is a challenging task. Instead of adopting the factor-graph-based equalizer structure like the MP [18], we investigate the structure and characteristics of OTFS transmission matrix, and design an iterative receiver, which targets the symbol detection and interference cancellation in the CoDSC.

We use (2) and (3) to formulate the OTFS transmission as the following matrix form,

$$
\mathbf{y}=\mathbf{G} \mathbf{x}+\mathbf{w}
$$

Vector $\mathbf{x} \in \mathbb{C}^{M N \times 1}$ denotes the transmitted symbol vector

$$
\mathbf{x}=\left[\mathbf{X}(0,:), \cdots, \mathbf{X}\left(l^{\prime},:\right), \cdots, \mathbf{X}(M-1,:)\right]^{T},
$$

The received symbol vector $\mathbf{y}$ and the noise vector $\mathbf{w}$ take the same form of $\mathbf{x}$. In $29, \mathbf{G} \in \mathbb{C}^{M N \times M N}$ denotes the channel gain matrix (CGM), or namely OTFS transmission matrix. According to [17], we have

$$
\mathbf{G}=\mathbf{B}\left(\mathbf{F}_{N} \otimes \mathbf{I}_{M}\right) \mathbf{H}_{\mathrm{TD}}\left(\mathbf{F}_{N}^{H} \otimes \mathbf{I}_{M}\right) \mathbf{B}^{H},
$$

${ }^{2}$ In this paper, the subcarrier spacing and the carrier frequency are $15 \mathrm{KHz}$ and $4 \mathrm{GHz}$, respectively. The maximum UE's speed is considered as 540 $\mathrm{km} / \mathrm{h}$, of which the maximum Doppler frequency normalized to the symbol rate $\tilde{f}_{d}$ is $15 \%$.

$$
\mathbf{G}=\left[\begin{array}{cccccccc}
\mathbf{G}_{0,0} & \mathbf{0} & \ldots & \mathbf{0} & \mathbf{G}_{L-1,0} & \ldots & \mathbf{G}_{2,0} & \mathbf{G}_{1,0} \\
\mathbf{G}_{1,1} & \mathbf{G}_{0,1} & \mathbf{0} & \cdots & \mathbf{0} & \mathbf{G}_{L-1,1} & \cdots & \mathbf{G}_{2,1} \\
\vdots & \vdots & \vdots & \vdots & \vdots & \vdots & \vdots & \vdots \\
\mathbf{0} & \cdots & \cdots & \mathbf{0} & \mathbf{G}_{L-1, M-2} & \cdots & \mathbf{G}_{0, M-2} & \mathbf{G}_{M-1, M-2} \\
\mathbf{0} & \cdots & \mathbf{0} & \mathbf{G}_{L-1, M-1} & \cdots & \cdots & \mathbf{G}_{1, M-1} & \mathbf{G}_{0, M-1}
\end{array}\right]
$$


where $\mathbf{H}_{\mathrm{TD}} \in \mathbb{C}^{M N \times M N}$ is the time-domain channel matrix constructed by the CIR samples, and $\mathbf{B}$ is an $M N \times M N$ permutation matrix [30]. Matrix $\mathbf{G}$ is blockbanded, shown in (32), where $\mathbf{G}_{\left(l-l^{\prime}\right)_{M}, l} \in \mathbb{C}^{N \times N} ; l, l^{\prime}=$ $0, \cdots, M-1$ is a submatrix. The element in the $v$-th row and $v^{\prime}$-th column of $\mathbf{G}_{\left(l-l^{\prime}\right)_{M}, l}$, is $\mathbf{G}_{\left(l-l^{\prime}\right)_{M}, l}\left(v, v^{\prime}\right)=$ $\Omega\left\{l,\left(l-l^{\prime}\right)_{M}, v-v^{\prime}\right\} ; v, v^{\prime}=0, \cdots, N-1$.

Fig. 3 intuitively demonstrates the structure of $\mathbf{G}$ and $\mathbf{G}_{\left(l-l^{\prime}\right)_{M}, l}$ in CoDSC, where the block size $M \times N=8 \times 8$ is considered. Subfigures (a) and (b) illustrate $\mathbf{G}$ and $\mathbf{G}_{\left(l-l^{\prime}\right)_{M}, l}$ in mathematics, respectively. The ISI is contributed by the submatrices $\mathbf{G}_{1, l}, \cdots, \mathbf{G}_{L-1, l}, l=0, \cdots, M-1$. When the channel's delay spread is zero, $\mathbf{G}_{\left(l-l^{\prime}\right)_{M}, l}=\mathbf{0} ; \forall\left(l-l^{\prime}\right)_{M} \neq$ 0 , CGM $\mathbf{G}$ turns into a block diagonal matrix and no ISI appears. Meanwhile, the IDI is contributed by the offdiagonal elements of $\mathbf{G}_{\left(l-l^{\prime}\right)_{M}, l}$ in (32). When the channel's Doppler spread becomes zero, implying the channel is static, submatrix $\mathbf{G}_{\left(l-l^{\prime}\right)_{M}, l} ; \forall l, l^{\prime}$ turns into a diagonal matrix and thus the IDI disappears. Subfigures (c) and (d) present the instances of matrices $\mathbf{G}$ and $\mathbf{G}_{\left(l-l^{\prime}\right)_{M}, l}$, respectively. As we can see, channel matrix $\mathbf{G}$ is block-banded. The number of non-zero submatrices in each row or column direction is $L-1$.

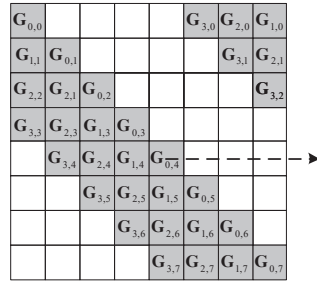

(a) Matrix $\mathbf{G}$

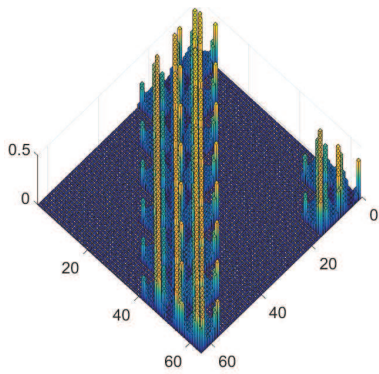

(c) An instance of $\mathbf{G}$.

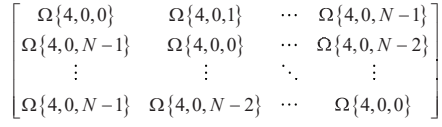

(b) Submatrix $\mathbf{G}_{\left(l-l^{\prime}\right)_{M}, l}$.

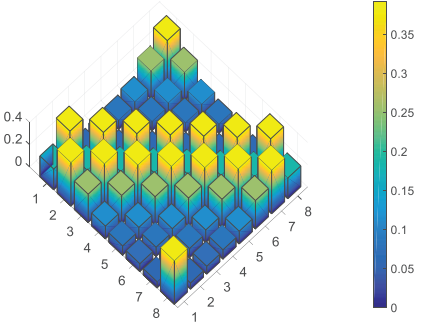

(d) An instance of $\mathbf{G}_{\left(l-l^{\prime}\right)_{M}, l}$.
Fig. 3. The structure of channel matrix in the case of CoDSC, where the maximum channel delay spread $L=4$.

To compare the IDI difference between LDSC and CoDSC, we plot some parts of the DD-domain channel responses of them in Fig. 4 As an example, we plot the first row of $\mathbf{G}_{0,4}(0,:)$ in Fig. 3(b). There are two Doppler shifts in the LDSC. Thus, in the Doppler-frequency direction, two major components are observed in Fig. 4(a). For the CoDSC, since the channel spectrum occupies a continuous range in the Doppler frequency, the bandwidth of $\Omega\left(4,0, v^{\prime}\right)$ for $\left|\Omega\left(4,0, v^{\prime}\right)\right|>0.1$, are much wider than that in the LDSC.

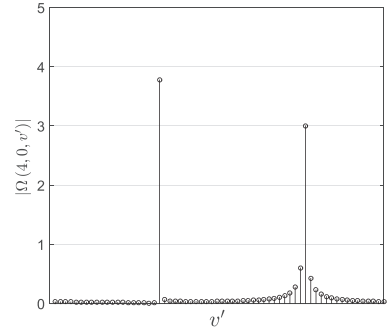

(a) An instance of $\left|\Omega\left(4,0, v^{\prime}\right)\right|$ in the LDSC

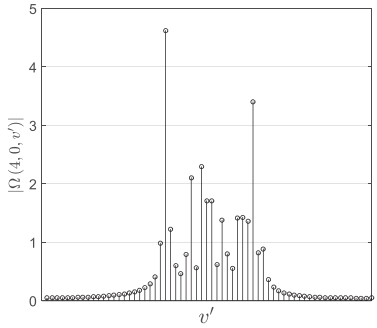

(b) An instance of $\left|\Omega\left(4,0, v^{\prime}\right)\right|$ in the CoDSC
Fig. 4. The illustration of $\Omega\left(4,0, v^{\prime}\right) ; v^{\prime}=0, \cdots, 63$, in the LDSC and CoDSC. There are two Doppler shifts in the LDSC. In the CoDSC, the maximum Doppler frequency normalized to the symbol rate $\tilde{f}_{d}$ is $15 \%$.

\section{A. Linear Equalizers}

The linear equalization methods, i.e, linear minimum mean square error (LMMSE) and zero forcing (ZF), are commonly employed to retrieve $\mathbf{x}$ from (29), expressed as

$$
\begin{gathered}
\hat{\mathbf{x}}_{\mathrm{LMMSE}}=\left(\mathbf{G}^{H} \mathbf{G}+\sigma^{2} \mathbf{I}\right)^{-1} \mathbf{G}^{H} \mathbf{y}, \\
=\left(\mathbf{G}^{H} \mathbf{G}\right)^{-1} \mathbf{G}^{H} \mathbf{y} .
\end{gathered}
$$

In the SDSC, matrix $\mathbf{G}$ is block-circulant [24]. Relying on the matrix-decomposition techniques, proposed in the previous OTFS receivers [24], [25], the implementation complexity of the LMMSE or ZF equalizer can be reduced from $\mathcal{O}\left(M^{3} N^{3}\right)$ to $\mathcal{O}(M N \log (M N))$. However, considering the OTFS signal transmission over CoDSC, matrix $\mathbf{G}$ is no longer block circulant. Accordingly, the low-complexity schemes can not be directly implemented. Due to the indispensable matrix inversion, we have to face a calculational complexity of $\mathcal{O}\left(M^{3} N^{3}\right)$ for realizing the LMMSE or ZF equalizes ${ }^{3}$

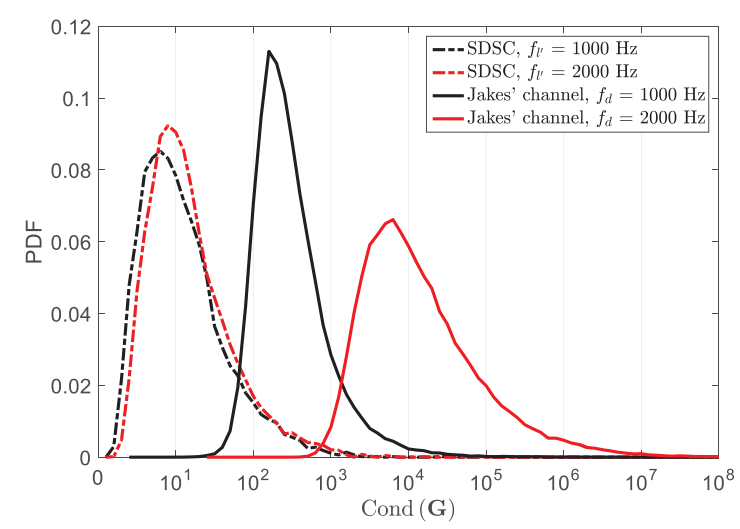

Fig. 5. The probability distribution function (PDF) of Cond (G).

When a CoDSC changes quickly, there is a high probability that matrix $\mathbf{G}$ is ill-conditioned ${ }^{4}$ i.e., $\operatorname{Cond}(\mathbf{G})$

\footnotetext{
${ }^{3}$ There are some matrix decomposition techniques, such as triangle decomposition and singular value decomposition |31|, can be used to reduce the complexity. However, the techniques still remain a complexity order of $\mathcal{O}\left(M^{2} N^{2}\right)$

${ }^{4}$ Whether $\mathbf{G}$ is ill-conditioned depends on its the condition number: Cond $(\mathbf{G})$. Since $\mathbf{B}\left(\mathbf{F}_{N} \otimes \mathbf{I}_{M}\right)$ and $\left(\mathbf{F}_{N}^{H} \otimes \mathbf{I}_{M}\right) \mathbf{B}^{H}$ in 31 are unitary matrices, we have Cond $(\mathbf{G})=$ Cond $\left(\mathbf{H}_{\mathrm{TD}}\right)$. According to [32 - [34], when the maximum Doppler frequency $f_{d}$ in the CoDSC is large, the value of Cond $\left(\mathbf{H}_{\mathrm{TD}}\right)$ fluctuates significantly and mostly concentrates in a large numerical interval. Hence, $\mathbf{H}_{\mathrm{TD}}$ is probably ill-conditioned.
} 


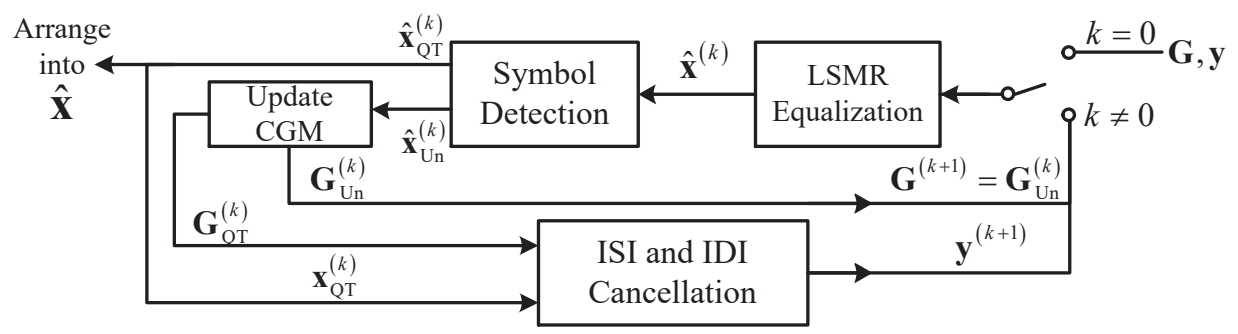

Fig. 6. The block diagram of the symbol detection and joint interference cancellation.

becomes quite large. To illustrate this, we give Fig. 5 as an example to show the probability distribution function (PDF) of Cond (G). In the Jakes' channel, the distribution of Cond $(\mathbf{G})$ is much broader than that in the $\mathrm{SDSC}^{5}$. Thus, for the Jakes' channel, the inversion of channel matrix may not robust enough, thereby introducing a significant equalization error in the LMMSE or ZF.

\section{B. The Proposed Iterative OTFS Receiver}

Recalling 29], the channel equalization is developed by solving the following LS problem

$$
\min _{\hat{\mathbf{x}} \in \mathbb{C}^{M N \times 1}}\|\mathbf{G} \hat{\mathbf{x}}-\mathbf{y}\|_{2} .
$$

The main features of $\mathbf{G}$ can be summarized: 1) the size of $\mathbf{G}$ is quite large for big OTFS transmission blocks; 2) matrix $\mathbf{G}$ is probably ill-conditioned, shown as Fig. 5, 3) it is a sparse matrix, shown as Fig. 3. Consequently, one-shot matrix inversion, adopted in the LMMSE and ZF equalizers, may result in significant performance degradation of the OTFS receiver.

In mathematics, some well-known iterative methods, such as LSMR [35], minimal residual (MINRES) [36] and leastsquares QR decomposition (LSQR) [37], were developed to solve large-scale, ill-conditioned and sparse LS problems as (35). The methods use a recursive strategy to obtain the LS solution with precision similar as the LMMSE method. Meanwhile, by exploiting the sparsity of $\mathbf{G}$, the iteration process needs much lower computational overhead. The LSMR iteration converges much faster than the LSQR. Also, the LSMR is algebraically equivalent to MINRES in solving the normal equation

$$
\left(\mathbf{G}^{H} \mathbf{G}+\sigma^{2} \mathbf{I}\right) \mathbf{x}=\mathbf{G}^{H} \mathbf{y},
$$

but has better numerical stability.

In this paper, focusing on the exclusive nature of OTFS transmission, we construct an LSMR based channel equalizer to remove the channel distortions on the transmitted OTFS symbols. Instead of using the conventionally hard decision on the symbol estimates from the channel equalizer, we propose a dynamic detection scheme to classify the symbol estimates into reliable and unreliable sets. In other words, we only detect the partial symbol estimates with high reliability to help

\footnotetext{
${ }^{5}$ The results are depicted by simulations. The entries in $\mathbf{G}$ are filled by the DD-domain channel gains, i.e., $\Omega\left\{l,\left(l-l^{\prime}\right)_{M}, v-v^{\prime}\right\}$, which are generated by the CIRs in (3). The CIRs are obtained by the SDSC and Jakes' channel simulators.
}

calculate the symbol introduced interference. Furthermore, a block-wise interference eliminator, i.e., a cascade of the symbol detector, is designed to realize the joint ISI and IDI cancellation. The interference eliminator contributes to modifying the observed data by removing the ISI and IDI caused by the detected symbols from the reliable sets. As a result, it helps to improve the accuracy of data detection in the next iteration 6

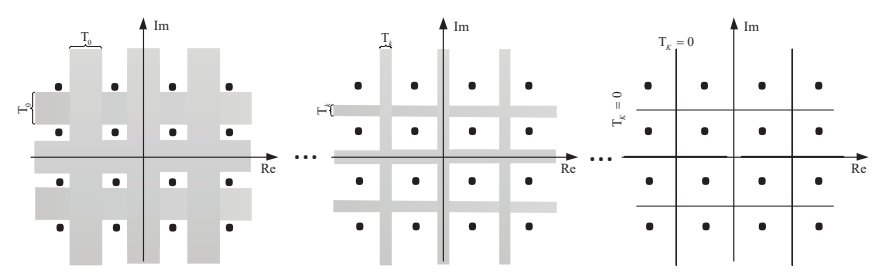

Fig. 7. Illustration of "unreliable zone" for the constellation of 16-QAM.

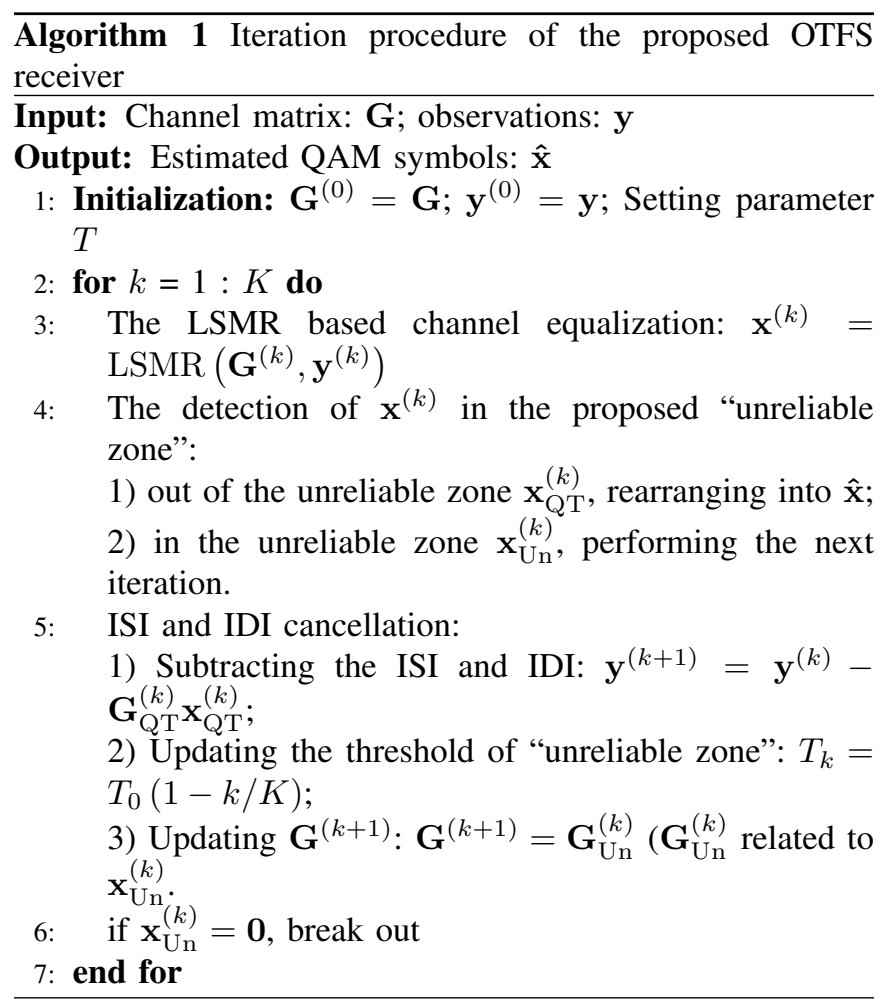

${ }^{6}$ Note that there are two types of iterations: 1) The iterations inside the LSMR based channel equalizer, to acquire the OTFS symbol estimates; 2) The iterations between the OTFS symbol detector and interference eliminator, to realize joint ISI and IDI cancellation. 
The proposed iterative symbol detection and interference cancellation for OTFS is illustrated in Fig. 6. In the $k$-th iteration, the LSMR aims to solve the overdetermined LS problem as

$$
\min _{\hat{\mathbf{x}}^{(k)}}\left\|\mathbf{G}^{(k)} \hat{\mathbf{x}}^{(k)}-\mathbf{y}^{(k)}\right\| .
$$

The output of the LSMR channel equalizer, i.e., the estimated symbols in $\hat{\mathbf{x}}^{(k)}$, will be detected in the proposed detection (quantization) zone with a dynamic threshold, as presented in Fig. 7. In this way, the data symbols in $\hat{\mathbf{x}}^{(k)}$ are divided into two subsets, which are composed of reliable symbols in $\hat{\mathbf{x}}_{\mathrm{QT}}^{(k)}$ and unreliable symbols in $\hat{\mathbf{x}}_{\mathrm{Un}}^{(k)}$, respectively. Then, the observation vector $\mathbf{y}^{(k)}$ will be updated through subtracting the ISI and IDI reconstructed by the reliable (quantized) symbols: $\mathbf{y}^{(k+1)}=\mathbf{y}^{(k)}-\mathbf{G}_{\mathrm{QT}}^{(k)} \mathbf{x}_{\mathrm{QT}}^{(k)}$, where $\mathbf{G}_{\mathrm{QT}}^{(k)}$ is formed by the columns of $\mathbf{G}^{(k)}$ corresponding to the reliable symbols in $\hat{\mathbf{x}}_{\mathrm{QT}}^{(k)}$. This step is also denoted as the joint ISI and IDI cancellation. Specifically, the iteration process is summarized in Algorithm 1. In the initial step, the observation vector and CGM are denoted as $\mathbf{y}^{(0)}=\mathbf{y} \in \mathbb{C}^{M N \times 1}$ and $\mathbf{G}^{(0)}=\mathbf{G} \in$ $\mathbb{C}^{M N \times M N}$, respectively. The width of the "unreliable zone" is set as $T_{0}$, as demonstrated in Fig. 7. The iteration process is described in the following part.

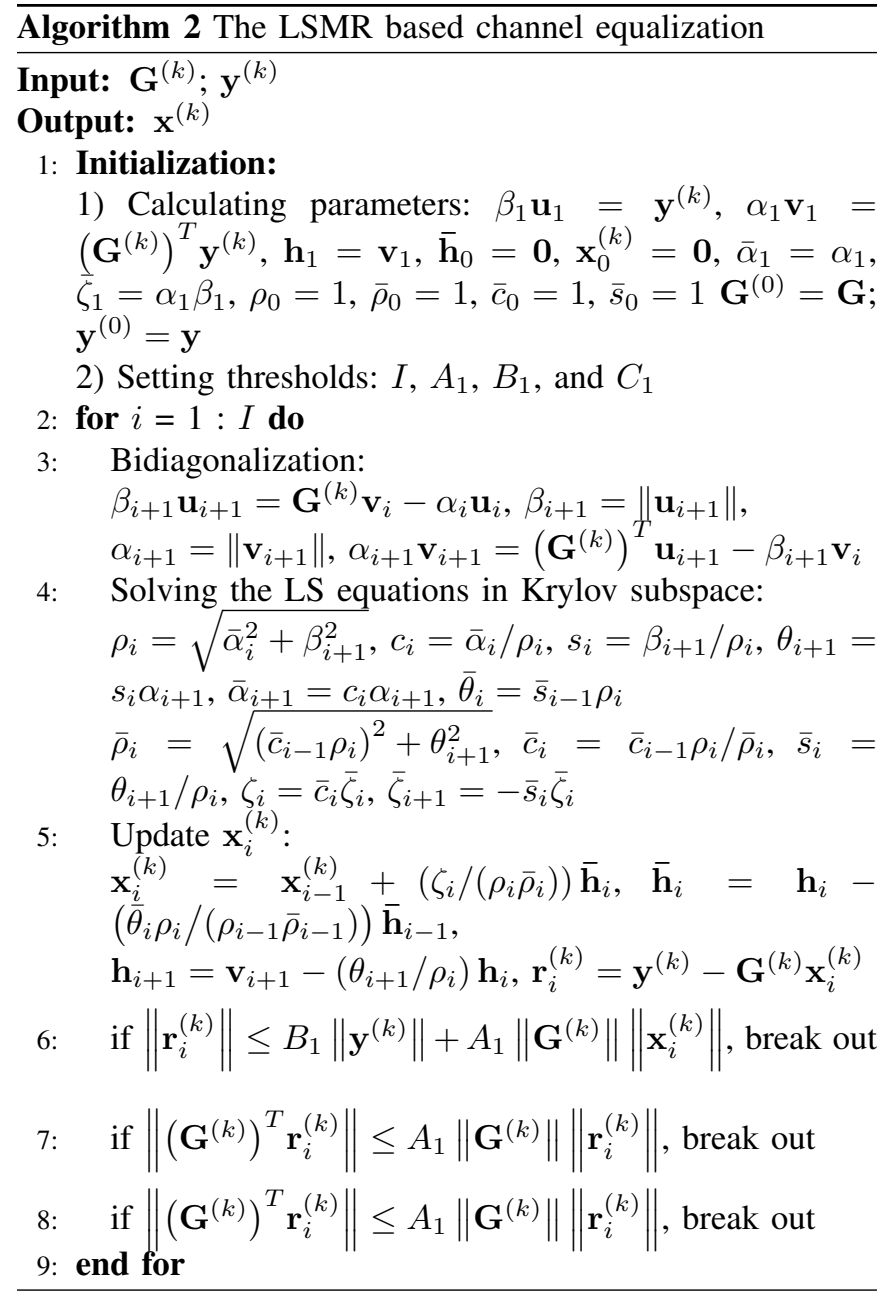

LSMR channel equalizer: In the $k$-th iteration, the observation vector $\mathbf{y}^{(k)} \in \mathbb{C}^{M N \times 1}$ and CGM G $\mathbf{G}^{(k)} \in \mathbb{C}^{M N \times J_{k}}$ are sent into the LSMR equalizer to estimate vector $\mathbf{x}^{(k)} \in$ $\mathbb{C}^{J_{k} \times 1}$, which is detailed in Algorithm 2. It begins with the Golub-Kahan process (Bidiagonalization) [38]. After the Bidiagonalization operation, the Krylov subspace $\mathbf{V}_{i}^{(k)}=$ $\left[\mathbf{v}_{1}, \mathbf{v}_{2}, \cdots, \mathbf{v}_{i}\right]$ is constructed to obtain $\mathbf{s}^{(k)}$ by solving the linear equation $\mathbf{x}_{i}^{(k)}=\mathbf{V}_{i}^{(k)} \mathbf{s}^{(k)}$. The estimated data vector $\mathbf{x}_{i}^{(k)}$ is further updated in the Krylov subspace. To accelerate the convergence in the iteration process, four stop criterions exist in the LSMR, as presented in Algorithm 2. Parameters $I, A_{1}, B_{1}$ and $C_{1}$ denote the iteration times, the error threshold of $\left\|\mathbf{G}^{(k)}\right\|\left\|\mathbf{x}_{i}^{(k)}\right\|$, the error threshold of $\left\|\mathbf{y}^{(k)}\right\|$ and the threshold of Cond $\left(\mathbf{G}^{(k)}\right)$, respectively. The quantities of $\left(\mathbf{G}^{(k)}\right)^{H} \mathbf{r}_{i}^{(k)}$ and $\mathbf{r}_{i}^{(k)}$ decrease monotonically in LSMR, where $\mathbf{r}_{i}^{(k)}=\mathbf{y}^{(k)}-\mathbf{G}^{(k)} \mathbf{x}_{i}^{(k)}$ is the residual for the approximate solution $\mathbf{x}_{i}^{(k)}$. For LSQR, only $\mathbf{r}_{i}^{(k)}$ decreases monotonically. Hence, LSQR is recommended for a compatible system $\mathbf{y}^{(k)}=\mathbf{G}^{(k)} \mathbf{x}_{i}^{(k)}$, but on LS problems with loose stopping tolerances, LSMR may be able to terminate significantly sooner than LSQR.

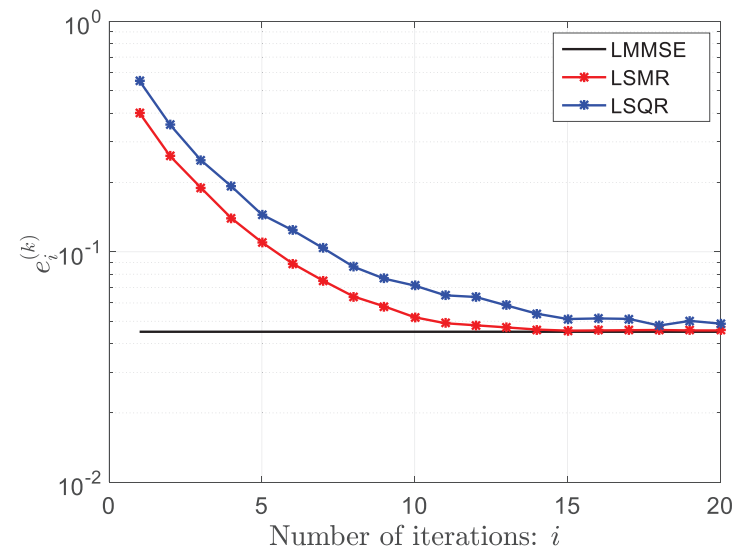

Fig. 8. The comparison of the estimation error $e_{i}^{(k)}$ versus the number of iterations $i$, where $\sigma^{2}=0.01$

We denote

$$
e_{i}^{(k)}=\mathbb{E}\left\{\left\|\mathbf{x}^{(k)}-\hat{\mathbf{x}}_{i}^{(k)}\right\|^{2}\right\}
$$

as the estimation error after the $i$-th iteration. Fig. 8 compares $e_{i}^{(k)}$ versus number of iterations $i$, where noise variance $\sigma^{2}=0.01$. The black line denotes the estimation error corresponding to the LMMSE algorithm, which is regarded as a threshold to evaluate the convergence of the LSMR and LSQR. It can be seen that as $i$ increases, $e_{i}^{(k)}$ gradually approaches to the threshold. Also, the LSMR converges faster than the LSQR. In this example, after 12 iterations, the LSMR can achieve the comparable symbol estimation accuracy against the LMMSE.

Reliability-based symbol detection: In each iteration, the equalized data symbols in $\hat{\mathbf{x}}^{(k)}$, i.e., the output of the LSMR equalizer, are quantized (detected) by utilizing the proposed quantization criterion. Inspired by [39], we design an "unreliable zone" in QAM constellation plane. The width of the "unreliable zone" is denoted by the threshold parameter 
$T_{k}$. Fig. 7 gives an example to demonstrate the "unreliable zone" in the constellation of 16-QAM. When the estimated symbol in $\hat{\mathbf{x}}^{(k)}$ does not fall inside the "unreliable zone", it will be detected as the reliable symbol and quantized to the nearest QAM symbol. Otherwise, it will not be quantized. The unreliable symbols will be picked out and sent into "update CGM" module. After quantization operation, the overall elements in $\hat{\mathbf{x}}^{(k)}$ are separated into two sets, quantized symbols (out of "unreliable zone") $\hat{\mathbf{x}}_{\mathrm{QT}}^{(k)} \in \mathbb{C}^{\left(J_{k}-J_{k+1}\right) \times 1}$ and unreliable symbols (in the "unreliable zone") $\hat{\mathbf{x}}_{\mathrm{Un}}^{(k)} \in$ $\mathbb{C}^{J_{k+1} \times 1}$. To accelerate the convergence of our algorithm, the width of the unreliable zone decreases to $T_{k}=T_{0}(1-k / K)$ after each iteration, and finally equals zero so as to ensure the overall symbols can be quantized. Note that when $T_{k}=0$, the quantization criterion becomes a conventionally hard-decision metric on symbol estimates.

CGM update: In the $k$-th iteration, after quantization operation, the CGM will be updated. Specifically, $\mathbf{G}^{(k)} \in \mathbb{C}^{M N \times J_{k}}$ is divided into two submatrices, i.e. $\mathbf{G}_{\mathrm{Un}}^{(k)} \in \mathbb{C}^{M N \times J_{k+1}}$ and $\mathbf{G}_{\mathrm{QT}}^{(k)} \in \mathbb{C}^{M N \times\left(J_{k}-J_{k+1}\right)}$. Matrix $\mathbf{G}_{\mathrm{Un}}^{(k)}$ is composed of $J_{k+1}$ columns of $\mathbf{G}^{(k)}$ corresponding to unreliable symbols. It will be arranged into the LSMR equalizer as the updated CGM $\mathbf{G}^{(k+1)}=\mathbf{G}_{\mathrm{Un}}^{(k)}$, and further employed to perform the next iteration. Matrix $\mathbf{G}_{\mathrm{QT}}^{(k)}$ is formed by the $J_{k}-J_{k+1}$ columns of $\mathbf{G}^{(k)}$ corresponding to the quantized symbols in $\hat{\mathbf{x}}_{\mathrm{QT}}^{(k)}$. It will be sent into the interference eliminator, to reconstruct the ISI and IDI.

ISI and IDI cancellation: Relying on the detected data symbols $\hat{\mathbf{x}}_{\mathrm{QT}}^{(k)}$ and the corresponding CGM $\hat{\mathbf{x}}_{\mathrm{QT}}^{(k)}$, we can reconstruct the ISI and IDI as: $\mathbf{G}_{\mathrm{QT}}^{(k)} \hat{\mathbf{x}}_{\mathrm{QT}}^{(k)}$. The received data vector is updated through subtracting the ISI and IDI as $\mathbf{y}^{(k+1)}=\mathbf{y}^{(k)}-\mathbf{G}_{\mathrm{QT}}^{(k)} \hat{\mathbf{x}}_{\mathrm{QT}}^{(k)}$. It is a block-wise interference cancellation scheme. After that, the interference-eliminated observation vector $\mathbf{y}^{(k+1)}$ will be sent into the LSMR equalizer to perform the next iteration.

Remark 2: The proposed symbol detector, combined with the interference eliminator, can work efficiently in the OTFS receiver. On the one hand, the LMSR with the proposed quantization method can ideally retrieve a subset of the transmitted QAM symbols in each iteration. The estimated QAM symbols enables the corresponding ISI and IDI to be accurately reconstructed. On the other hand, the blockwise interference eliminator can remove the ISI and IDI from the received data vector with linear implementation complexity. The updated observation vector and CGM, also helps to improve the symbol estimation performance in the next iteration.

\section{Computational Complexity}

Here, we compare the computational complexity of three detection schemes, i.e., LMMSE, MP [18] and ours. For the LMMSE, the complexity order is $\mathcal{O}\left(M^{3} N^{3}\right)$, which only depends on the size of OTFS transmission block, i.e., $M N$. For the MP, the complexity order is $\mathcal{O}\left(M N B Q I_{M P}\right)$ [18], where $B, Q$ and $I_{M P}$ are the number of nonzero elements in each row of $\mathbf{G}$, alphabet size and iteration times, respectively. In the proposed scheme, the complexity order of implementing the LSMR is $\mathcal{O}(M N B I)$ [35]. With $K$ iterations, the overall complexity order is $\mathcal{O}(M N B I K)$. In this paper, we suppose the channel gains occupy the whole grids in the Doppler dimension, which follows $B=N L$. Hence, the complexity orders of the proposed and MP schemes are $\mathcal{O}\left(M N^{2} L I K\right)$ and $\mathcal{O}\left(M N^{2} L Q I_{M P}\right)$, respectively. It should be noted that the complexity of the proposed algorithm relates to the OTFS transmission block size $(M, N)$, but is independent of the modulation order $Q$.

The difference between ours and the MP algorithms lies in the selection of parameters $I, K, I_{M P}$ and $Q$. In general, the number of iteration times in our algorithm is much fewer than the MP, i.e., $K \ll I_{M P}$. Taking 16-QAM signaling as an example, the typical values are: $I=15, K=10$, $I_{M P}=50$ and $Q=16$. Thus, we have $I K \ll I_{M P} Q$, indicating much lower computation cost. In practice, for different QAM constellation sizes, parameters $I, K$ and $I_{M P}$ will be properly chosen to achieve a tradeoff between the computation overhead and demodulation performance.

\section{Simulation Results}

In this section, the demodulation performance is evaluated for the OTFS system, of which the parameters are set according to Tab. I.

TABLE I

OTFS SySTEM SIMULATION PARAMETERS

\begin{tabular}{|c|c|}
\hline \hline Parameter & Value \\
\hline Num. of subcarriers $(M)$ & 64 \\
\hline Length of CP $\left(M_{c p}\right)$ & 8 \\
\hline Num. of symbols $(N)$ & $6 / 12 / 24$ \\
\hline Carrier frequency $\left(f_{c}\right)$ & $4 \mathrm{GHz}$ \\
\hline Subcarrier spacing $(\Delta f)$ & $15 \mathrm{KHz}$ \\
\hline UE's velocity & $2.7 \sim 675 \mathrm{~km} / \mathrm{h}$ \\
\hline Modulation scheme & QPSK, 16-QAM, 64-QAM \\
\hline Channel model & Extended vehicular A EVA) $\mid 40$ \\
\hline Channel Doppler spectrum & Continuous "U'-shaped spectrum $\mid 28$ \\
\hline \hline
\end{tabular}

In the channel simulator, the channel taps are assumed to be independently and identically distributed random variables correlated in time according to (33) with maximum Doppler frequency $f_{d}[41]$. To avoid the detection performance loss introduced by channel estimation errors, we assume that the channel responses are perfectly known at the receiver side. In practice, the channel can be precisely estimated by using the methods in [42]. Both the uncoded and coded demodulation performance are investigated in this section. For the uncoded case, we present the symbol error rate (SER) results to focus on the demodulation performance. In the coded performance simulation, a recursive systematic convolutional code with the coding rate of $2 / 3$ and a random interleaver are employed.

We consider some scenarios with $f_{d}$ ranging from $10 \mathrm{~Hz}$ to $2500 \mathrm{~Hz}$, corresponding the velocities from $2.7 \mathrm{~km} / \mathrm{h}$ to $675 \mathrm{~km} / \mathrm{h}$. Note that the proposed OTFS framework can be potentially applied to wireless transmissions with large number of surroundings, such as V2X communications [43], [44]. Additionally, the widely-used equalizers, i.e., the 


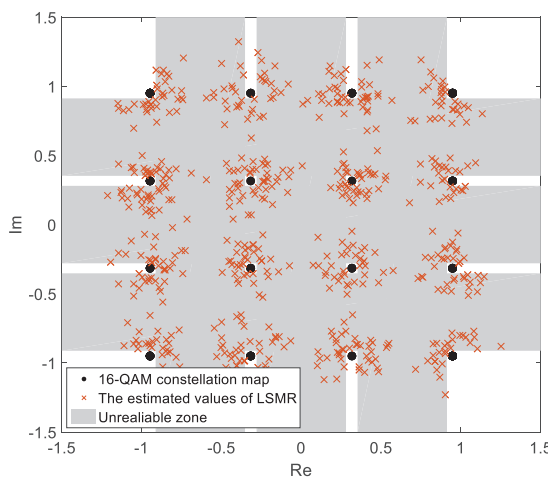

(a) The initial step, $k=0$.

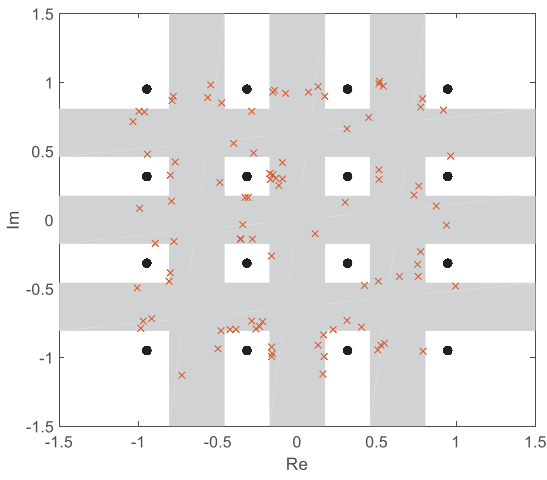

(d) The 6th iteration, $k=6$.

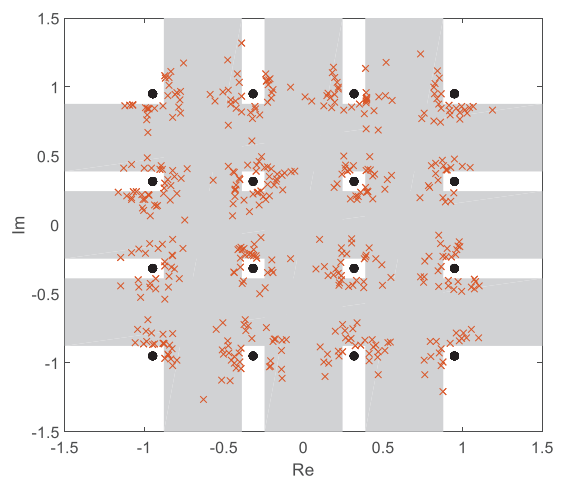

(b) The 2nd iteration, $k=2$.

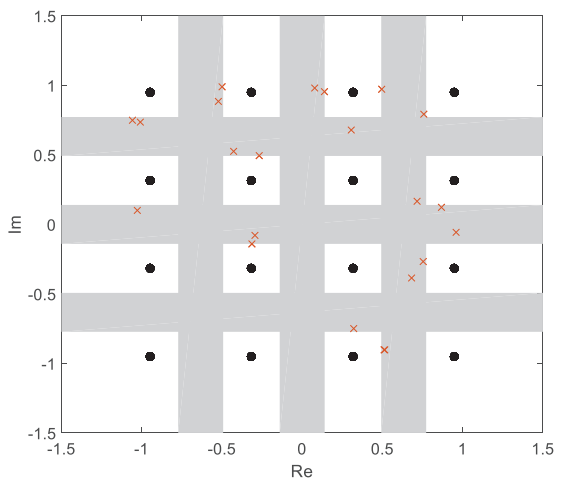

(e) The 8 th iteration, $k=8$.

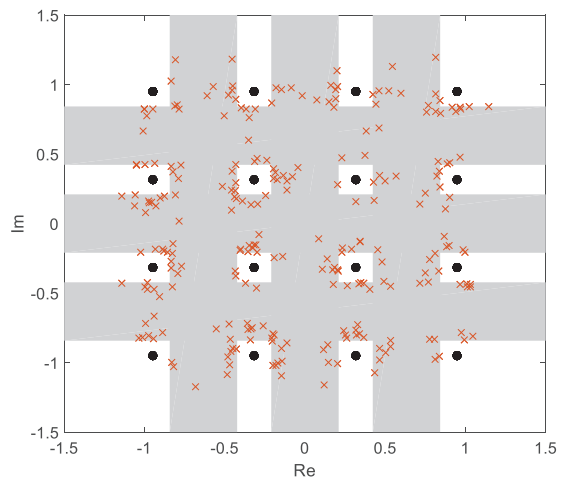

(c) The 4th iteration, $k=4$.

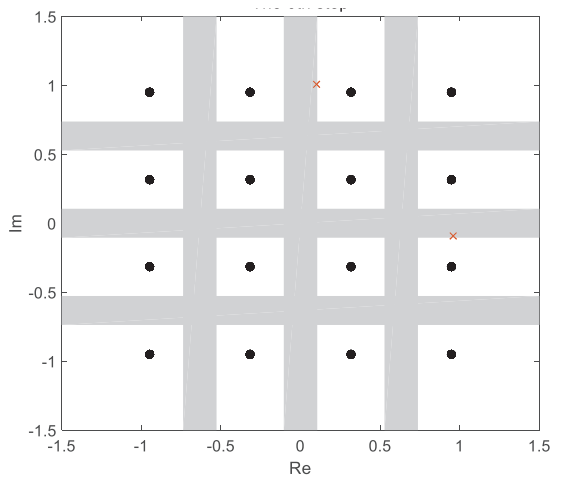

(f) The 10th iteration, $k=10$.

Fig. 9. The distribution of unreliable symbols after $k$-th iteration, when $\mathrm{SNR}=20 \mathrm{~dB}$.

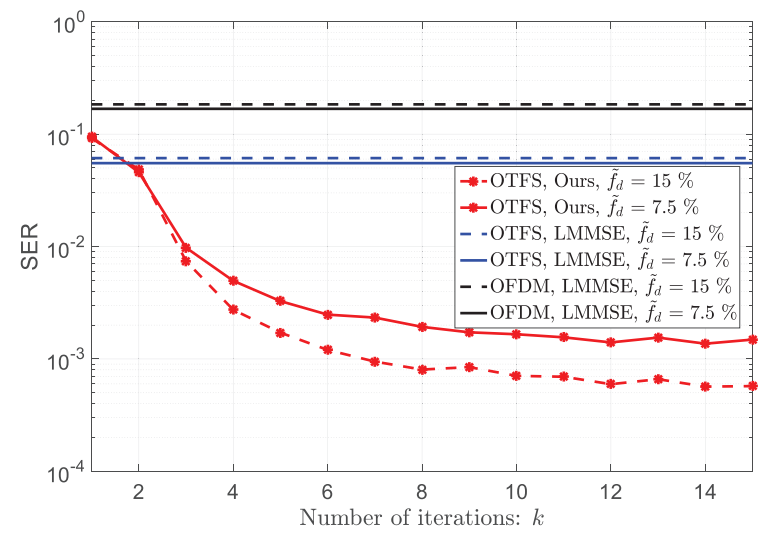

Fig. 10. The SER versus the number of iterations $k$ for 16-QAM, when $\mathrm{SNR}=20 \mathrm{~dB}$.

LMMSE [24] and the MP [18], are compared in terms of SER performance and implementation complexity.

The convergence process of the proposed algorithm is shown in Fig. 9, through the scatterplots of constellations after iterations. In each subfigure, the black points and the gray region denote the symbols in 16-QAM alphabet and the "unreliable zone", respectively. When $k \leq 4$, there is a relatively large number of unreliable symbols in the grey zone. As $k$ increases, the region of "unreliable zone" shrinks, and more LSMR estimates are quantized (detected) as the reliable symbols. As we can see, the proposed symbol

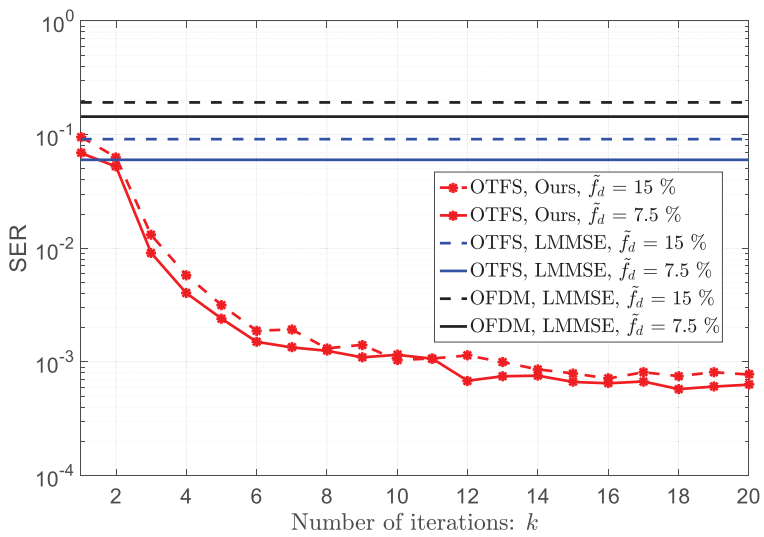

Fig. 11. The SER versus the number of iterations $k$ for 64-QAM, when $\mathrm{SNR}=28 \mathrm{~dB}$

detection scheme can achieve a desired convergence rate. After 10 iterations, there are only two unreliable symbols remaining in the grey zone.

Figs. 10 and 11 compare the SER versus the number of iterations $k$ for 16-QAM and 64-QAM signaling, respectively. It can be seen that the OTFS achieves the superior SER performance to OFDM in both the moderate- and high-Dopplerspreading scenarios. When $k=0$, the joint ISI and IDI elimination scheme is not invoked, while the one-shot LSMR equalizer has the similar SER performance to the LMMSE equalizer. After ten iterations, a significant SER performance 


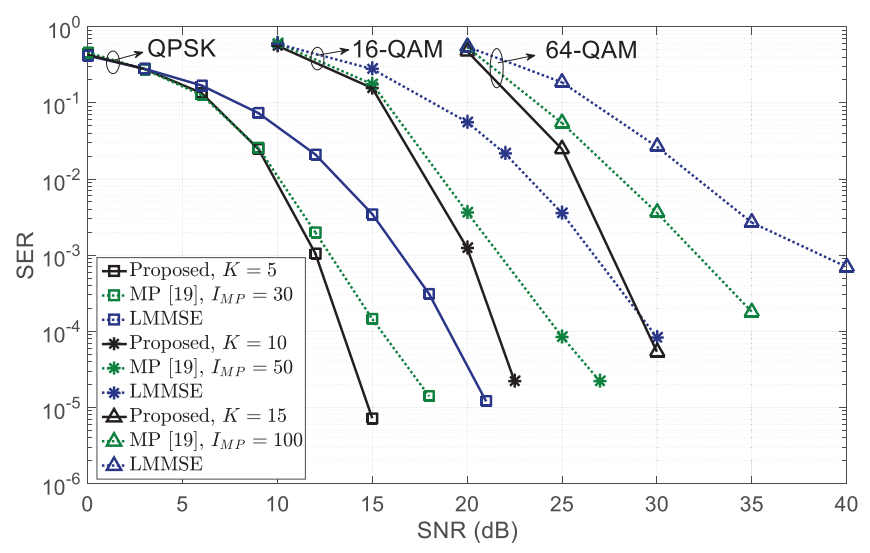

Fig. 12. The SER comparison of SNR, when $f_{d}=1000 \mathrm{~Hz}$ (corresponding to a velocity of $270 \mathrm{~km} / \mathrm{h}$ ).

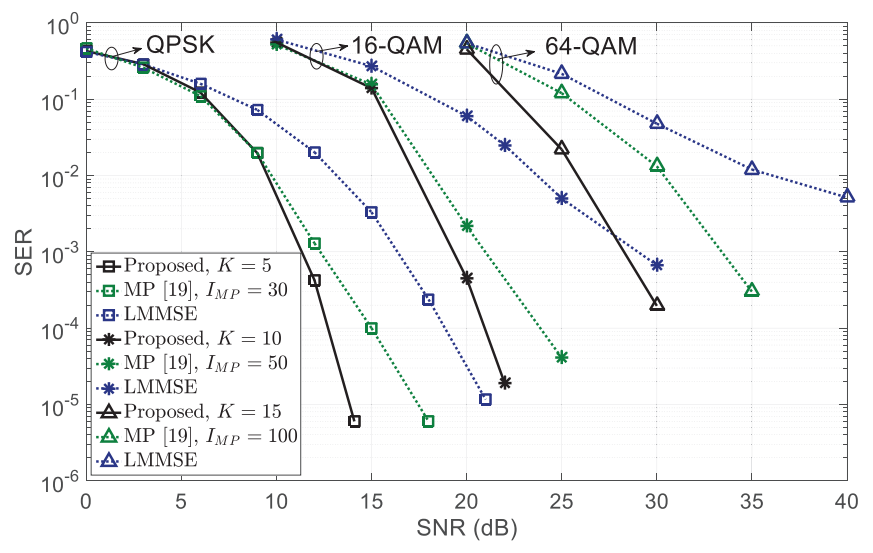

Fig. 13. The SER comparison of SNR, when $f_{d}=2000 \mathrm{~Hz}$ (corresponding to a velocity of $540 \mathrm{~km} / \mathrm{h}$ ).

improvement is achieved for the proposed OTFS receiver. Therefore, we choose the maximum number of iterations $K$ as 10 for 16-QAM signaling, to tradeoff the performance and complexity. For 64-QAM, as shown in Fig. 11, $K$ is chosen as 15 .

Figs. 12 and 13 compare the SER versus SNR for different modulation schemes in velocities of 270 and $540 \mathrm{~km} / \mathrm{h}$, respectively. For the MP based detection [18], the iteration times for QPSK, 16-QAM and 64-QAM are chosen as $I_{M P}$ $=30,50$, and 100, respectively, for obtaining stable SER results. In contrast, for these constellations, the iteration times of the proposed scheme can be set as $K=5,10$, and 15, respectively. Meanwhile, the developed receiver significantly outperforms the LMMSE receiver. As shown in Fig. 12. for SER $=2 \times 10^{-4}$, the achievable SNR gain is about $8 \mathrm{~dB}$ for 16-QAM signaling. Further, compared with the nonlinear receiver using the MP scheme, the SNR gains are about $1.6 \mathrm{~dB}, 3 \mathrm{~dB}$ and $6 \mathrm{~dB}$ for QPSK, 16-QAM and 64QAM signaling, respectively. Similarly, in Fig. 13, for SER = $3 \times 10^{-4}$, the proposed receiver can achieve about $1.5 \mathrm{~dB}, 2.3$ $\mathrm{dB}$ and 5.5 dB SNR gains for QPSK, 16-QAM and 64-QAM signaling, respectively, in contrast to the MP based receiver [18].

To evaluate the equalization performance under the differ-

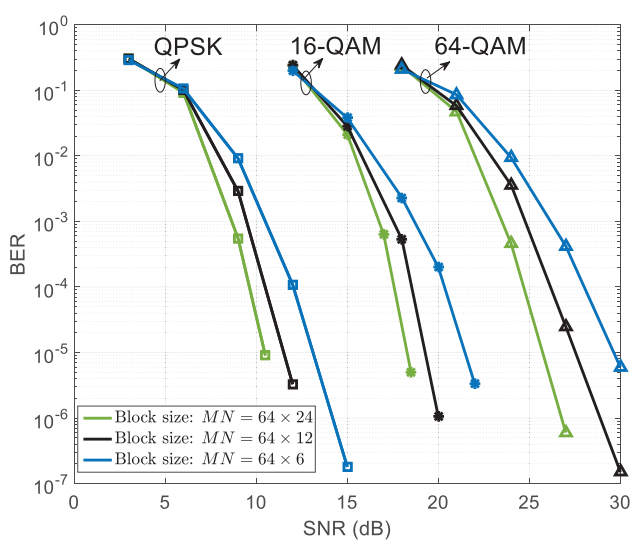

Fig. 14. The coded BER performance of the proposed receiver with different OTFS block sizes and QAM constellations, when $f_{d}=2000 \mathrm{~Hz}$.
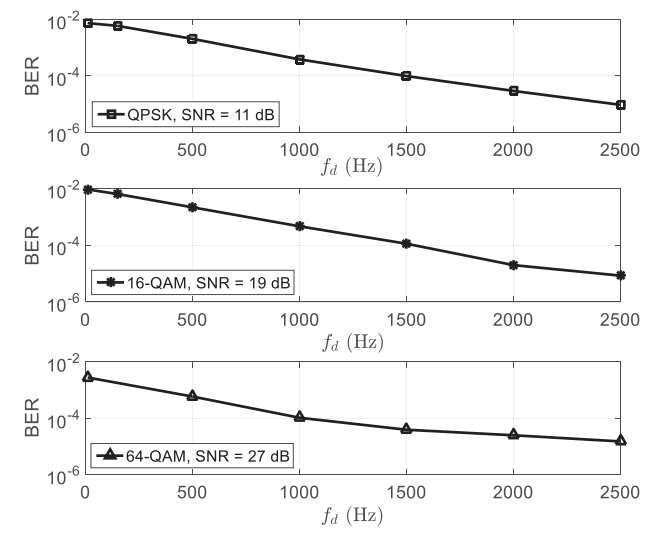

Fig. 15. The BER versus $f_{d}$ in coded OTFS system, where the block size is $M \times N=64 \times 12$. The SNRs for QPSK, 16-QAM, and 64-QAM signaling are $11 \mathrm{~dB}, 19 \mathrm{~dB}$, and $27 \mathrm{~dB}$, respectively.

ent traffic loads, we simulate the coded bit error rate (BER) performance as Fig. 14. Given $M \times N=64 \times 12$, the SNR gap among QPSK, 16-QAM and 64-QAM signaling is about $8.5 \mathrm{~dB}$ at $\mathrm{BER}=1 \times 10^{-5}$. In addition, for a certain QAM constellation, increasing the OTFS block size, which yields an enhanced sampling resolution in Doppler frequency, results in an improved BER performance.

Fig. 15 demonstrates the BER floor versus $f_{d}$ in the coded OTFS system. As shown in Fig. 15, the BER floors decrease as $f_{d}$ increases. An encouraging conclusion, hence, can be reached, that the stronger IDI cannot deteriorate the performance of the designed equalizer, but improve it. The reason is that the interference is treated as channel diversity in our receiver and the corresponding gains can be obtained for enhancing the demodulation performance in high mobilities.

In the end, the computational complexity in terms of complex multiplications (CMs) is illustrated in Fig. 16. We calculate the number of CMs according to Sec. IV-C. The corresponding parameters are consistent with those used in Figs. 12 and 13. It can be seen that for all the considered QAM constellations, the proposed algorithm achieves the lowest computation overhead, while the LMMSE has the highest complexity. In contrast to the MP, the CMs of our scheme can be decreased by 2, 4.5, and 17.9 times for QPSK, 
16-QAM and 64-QAM, respectively.

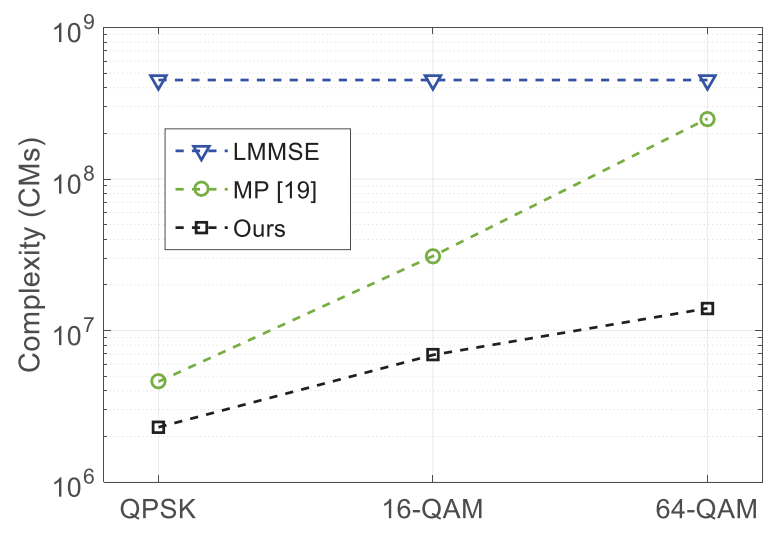

Fig. 16. The comparison of computational complexity.

\section{Conclusions}

In this paper, we study the orthogonal time frequency space (OTFS) signal transmission over the continuous-Dopplerspread channel (CoDSC) with the focus on the symbol detection and interference cancellation. Specifically, we analyze the generation mechanism and distribution of the inter-Doppler interference, and find that the factor-graph based message passing equalizer in the state-of-the-art works is not efficient to cope with the CoDSC incurred interference. According to the characteristics of the OTFS transmission matrix, we propose a low-complexity equalizer utilizing the mathematical least squares minimum residual (LSMR) algorithm. Based on the LSMR equalization, we develop an iterative OTFS receiver by introducing the reliability-based dynamic detector and block-wise interference eliminator. The simulations demonstrate that the developed iteration scheme, between the symbol detection and interference cancellation, outperforms the state-of-the-art OTFS demodulation schemes with respect to error-rate performance and computation complexity.

\section{APPENDIX A ProOF OF Proposition 1}

The transmitted time-domain data block in OTFS is formulated as

$$
\mathbf{S}=\mathbf{F}_{M}^{H} \mathbf{D}=\mathbf{X F}_{N}^{H}
$$

At the receiver side, after removing $\mathrm{CP}$, the $i$-th received vector $\mathbf{r}_{i} \in \mathbb{C}^{M \times 1}$ is represented as $\mathbf{r}_{i}=\mathbf{H}_{i} \mathbf{s}_{i}+\breve{\mathbf{w}}_{i}$, where $\mathbf{s}_{i} \in \mathbb{C}^{M \times 1}$ is the $i$-th column vector of $\mathbf{S}, \overline{\mathbf{w}}_{i} \in \mathbb{C}^{M \times 1}$ denotes the AWGN vector and $\mathbf{H}_{i} \in \mathbb{C}^{M \times M}$ is a Toeplitz matrix constructed by CIR values. The DD-domain received data block $\mathbf{Y}$ is expressed as

$$
\begin{aligned}
\mathbf{Y} & =\mathbf{F}_{M}^{H} \tilde{\mathbf{D}} \mathbf{F}_{N}=\mathbf{R} \mathbf{F}_{N} \\
& =\left[\mathbf{H}_{0} \mathbf{s}_{0}, \cdots, \mathbf{H}_{N-1} \mathbf{s}_{N-1}\right] \mathbf{F}_{N}+\mathbf{W},
\end{aligned}
$$

where $\mathbf{W}=\left[\breve{\mathbf{w}}_{0}, \cdots, \breve{\mathbf{w}}_{N-1}\right] \mathbf{F}_{N}$. The element at the $l$-th row and the $v$-th column of $\mathbf{Y}$, denoted as $\mathbf{y}_{v}(l)$, is expressed in (39), where $H_{i}\left(l, l^{\prime}\right)$ denotes the $l$-th row and $l^{\prime}$-th column of $\mathbf{H}_{i}$, i.e.,

$$
H_{i}\left(l, l^{\prime}\right)=h\left[i\left(M+M_{c p}\right)+M_{c p}+l,\left(l-l^{\prime}\right)_{M}\right]
$$

and $\mathbf{x}_{v^{\prime}}\left(l^{\prime}\right)$ and $\mathbf{w}_{v}\left(l^{\prime}\right)$ take the same form of $\mathbf{y}_{v}(l)$. Defining the DD-domain channel response $\Omega\left\{l,\left(l-l^{\prime}\right)_{M}, v-v^{\prime}\right\}$ as (40) then we have

$$
Y(l, v)=\sum_{v^{\prime}=0}^{N-1} \sum_{l^{\prime}=0}^{M-1} \Omega\left\{l,\left(l-l^{\prime}\right)_{M}, v-v^{\prime}\right\} X\left(l^{\prime}, v^{\prime}\right)+W(l, v) \text {. }
$$

\section{REFERENCES}

[1] Y. Hu, Z. Chang, H. Li, T. Ristaniemi, and Z. Han, "Service provisioning and user association for heterogeneous wireless railway networks," IEEE Trans. Commun., vol. 65, no. 7, pp. 3066-3078, July 2017.

[2] J. Wu and P. Fan, "A survey on high mobility wireless communications: Challenges, opportunities and solutions," IEEE Access, vol. 4, pp. 450476, Jan. 2016.

[3] 3GPP, Study on enhancement of 3GPP Support for $5 G$ V2X Services, 3GPP Release 15, TR 22.886 V15.1.0 Std., 2017.

[4] H. Jiang, Z. Zhang, J. Dang, and L. Wu, "A novel 3-D massive MIMO channel model for vehicle-to-vehicle communication environments," IEEE Trans. Commun., vol. 66, no. 1, pp. 79-90, Jan. 2018.

[5] P. S. Bithas, A. G. Kanatas, D. B. da Costa, P. K. Upadhyay, and U. S. Dias, "On the double-generalized gamma statistics and their application to the performance analysis of $\mathrm{V} 2 \mathrm{~V}$ communications," IEEE Trans. Commun., vol. 66, no. 1, pp. 448-460, Jan. 2018.

[6] M. M. Azari, F. Rosas, K.-C. Chen, and S. Pollin, "Ultra reliable UAV communication using altitude and cooperation diversity," IEEE Trans. on Commun., vol. 66, no. 1, pp. 330-344, Aug. 2017.

[7] V. V. Chetlur and H. S. Dhillon, "Downlink coverage analysis for a finite 3-D wireless network of unmanned aerial vehicles," IEEE Trans. Commun., vol. 65, no. 10, pp. 4543-4558, Oct. 2017.

[8] R. Hadani and A. Monk, "OTFS: A new generation of modulation addressing the challenges of 5G," arXiv preprint: 1802.02623, 2018.

[9] P. Schniter, "Low-complexity equalization of OFDM in doubly selective channels," IEEE Trans. Signal Process., vol. 52, no. 4, pp. 1002-1011, Apr. 2004.

[10] Y.-S. Choi, P. J. Voltz, and F. A. Cassara, "On channel estimation and detection for multicarrier signals in fast and selective rayleigh fading channels," IEEE Trans. Commun., vol. 49, no. 8, pp. 1375-1387, Aug. 2001.

[11] G. Liu, S. V. Zhidkov, H. Li, L. Zeng, and Z. Wang, "Low-complexity iterative equalization for symbol-reconstruction-based OFDM receivers over doubly selective channels," IEEE Trans. Broadcast., vol. 58, no. 3, pp. 390-400, Sept. 2012.

$$
\begin{aligned}
& \mathbf{y}_{v}(l)=Y(l, v)=\frac{1}{N} \sum_{v^{\prime}=0}^{N-1} \sum_{i=0}^{N-1}\left(\sum_{l^{\prime}=0}^{M-1} H_{i}\left(l, l^{\prime}\right) \mathbf{x}_{v^{\prime}}\left(l^{\prime}\right)\right) e^{-j 2 \pi i\left(v-v^{\prime}\right) / N}+\mathbf{w}_{v}(l), \\
& \Omega\left\{l,\left(l-l^{\prime}\right)_{M}, v-v^{\prime}\right\}=\frac{1}{N} \sum_{i=0}^{N-1} e^{\frac{-j 2 \pi i\left(v-v^{\prime}\right)}{N}} h\left[i\left(M+M_{c p}\right)+M_{c p}+l,\left(l-l^{\prime}\right)_{M}\right],
\end{aligned}
$$


[12] R. Hadani, S. Rakib, M. Tsatsanis, A. Monk, A. J. Goldsmith, A. F. Molisch, and R. Calderbank, "Orthogonal time frequency space modulation," in Proc. IEEE Wireless Communications and Networking Conference (WCNC), Mar. 2017.

[13] R. M. Augustine, G. Surabhi, and A. Chockalingam, "Space-time coded OTFS modulation in high-Doppler channels," in Proc. IEEE 89th Vehicular Technology Conference (VTC2019-Spring), May 2019.

[14] A. Rezazadehreyhani, A. Farhang, M. Ji, R. R. Chen, and B. FarhangBoroujeny, "Analysis of discrete-time MIMO OFDM-based orthogonal time frequency space modulation," in Proc. IEEE International Conference on Communications (ICC), May 2018.

[15] P. Raviteja, K. T. Phan, and Y. Hong, "Embedded pilot-aided channel estimation for OTFS in delay-Doppler channels," IEEE Trans. Wireless Commun., vol. 68, no. 5, pp. 4906-4917, May 2019.

[16] G. Surabhi, R. M. Augustine, and A. Chockalingam, "On the diversity of uncoded OTFS modulation in doubly-dispersive channels," IEEE Trans. Wireless Commun., vol. 18, no. 6, pp. 3049-3063, June 2019.

[17] A. Farhang, A. Rezazadehreyhani, L. E. Doyle, and B. FarhangBoroujeny, "Low complexity modem structure for OFDM-based orthogonal time frequency space modulation," IEEE Wireless Commun. Lett., vol. 7, no. 3, pp. 344-347, Nov. 2017.

[18] P. Raviteja, P. K. T., H. Yi, and V. Emanuele, "Interference cancellation and iterative detection for orthogonal time frequency space modulation," IEEE Trans. Wireless Commun., vol. 17, no. 10, pp. 6501-6515, Aug. 2018.

[19] P. Raviteja, K. T. Phan, Q. Jin, Y. Hong, and E. Viterbo, "Lowcomplexity iterative detection for orthogonal time frequency space modulation," in Proc. IEEE Wireless Communications and Networking Conference (WCNC), Apr. 2018, pp. 1-6.

[20] L. Li, Y. Liang, P. Fan, and Y. Guan, "Low complexity detection algorithms for OTFS under rapidly time-varying channel," in Proc. IEEE 89th Vehicular Technology Conference (VTC2019-Spring), May 2019.

[21] T. Zemen, M. Hofer, D. Loeschenbrand, and C. Pacher, "Iterative detection for orthogonal precoding in doubly selective channels," in Proc. IEEE 29th Annual International Symposium on Personal, Indoor and Mobile Radio Communications (PIMRC), Sept. 2018.

[22] F. Long, K. Niu, C. Dong, and J. Lin, "Low complexity iterative LMMSE-PIC equalizer for OTFS," in Proc. IEEE International Conference on Communications (ICC), May 2019.

[23] L. Li, H. Wei, Y. Huang, Y. Yao, W. Ling, G. Chen, P. Li, and Y. Cai, "A simple two-stage equalizer with simplified orthogonal time frequency space modulation over rapidly time-varying channels," arXiv preprint arXiv:1709.02505, 2017.

[24] S. Tiwari, S. S. Das, and V. Rangamgari, "Low complexity LMMSE receiver for OTFS," IEEE Commun. Lett., vol. 23, no. 12, pp. 22052209, Dec. 2019.

[25] G. Surabhi and A. Chockalingam, "Low-complexity linear equalization for OTFS modulation," IEEE Commun. Lett., vol. 24, no. 2, pp. 330334, Feb. 2020.

[26] C. X. Wang, A. Ghazal, B. Ai, Y. Liu, and P. Fan, "Channel measurements and models for high-speed train communication systems: A survey." IEEE Commun. Surv. Tut., vol. 18, no. 2, pp. 974-987, Dec. 2016.

[27] 3GPP, Study on LTE-based V2X Services, Rel. 14, TR 36.885 (V14.0.0) Std., 2016.

[28] C. Xiao, Y. R. Zheng, and N. C. Beaulieu, "Second-order statistical properties of the WSS Jakes' fading channel simulator," IEEE Trans. Commun., vol. 50, no. 6, pp. 888-891, June 2002.

[29] Z. Ding, R. Schober, P. Fan, and H. V. Poor, "OTFS-NOMA: An efficient approach for exploiting heterogenous user mobility profiles," IEEE Trans. Commun., vol. 67, no. 11, pp. 7950-7965, Nov. 2019.

[30] J. Han, S. P. Chepuri, Q. Zhang, and G. Leus, "Iterative per-vector equalization for orthogonal signal-division multiplexing over timevarying underwater acoustic channels," IEEE Journal of Oceanic Engineering, vol. 44, no. 1, pp. 240-255, Jan. 2019.

[31] G. H. Golub and C. F. Van Loan, Matrix Computations, 4th ed. Johns Hopkins Univ. press, 2013.

[32] J. Tong, Q. Guo, S. Tong, J. Xi, and Y. Yu, "Condition numberconstrained matrix approximation with applications to signal estimation in communication systems," IEEE Signal Process. Lett., vol. 21, no. 8, pp. 990-993, Aug. 2014.

[33] Y. Rong, S. A. Vorobyov, and A. B. Gershman, "Robust linear receivers for multiaccess space-time block-coded mimo systems: a probabilistically constrained approach," IEEE J. Sel. Areas Commun., vol. 24, no. 8, pp. 1560-1570, Aug. 2006.
[34] O. Ledoit and M. Wolf, "A well-conditioned estimator for largedimensional covariance matrices," J. Multivar. Anal., vol. 88, no. 2, pp. 365-411, 2004.

[35] D. C.-L. Fong and M. Saunders, "LSMR: An iterative algorithm for sparse least-squares problems," SIAM J. Sci. Comput., vol. 33, no. 5, pp. 2950-2971, Oct. 2011.

[36] C. C. Paige and M. A. Saunders, "Solution of sparse indefinite systems of linear equations," SIAM Journal Numer. Anal., vol. 12, no. 4, pp. 617-629, 1972.

[37] — - "LSQR: An algorithm for sparse linear equations and sparse least squares," ACM Trans. Mathemat. Software, vol. 8, no. 1, pp. 43-71, 1982.

[38] G. Golub and W. Kahan, "Calculating the singular values and pseudoinverse of a matrix," Journal Soc. Indus. Appl. Math. Ser. B Number. Anal., vol. 2, no. 2, pp. 205-224, 1965.

[39] M. Chiani, "Introducing erasures in decision-feedback equalization to reduce error propagation," IEEE Trans. Commun., vol. 45, no. 7, pp. 757-760, July 1997.

[40] 3GPP, Base Station (BS) conformance testing, Rel. 15, TS 36.141 (V15.1.0) Std., 2017.

[41] W. C. Jakes and D. C. Cox, Microwave mobile communications. Wiley-IEEE Press, 1994.

[42] H. Qu, G. Liu, Y. Wang, Q. Chen, C. Yi, and J. Peng, "A timedomain approach to channel estimation and equalization for the SCFDM system," IEEE Trans. Broadcast., vol. 65, no. 4, pp. 713 - 726, Dec. 2019

[43] F. Mikael, D. Markus, A. Z. Jesus, B. Mate, A. Taimoor, M. Konstantinos, M. Toktam, S. Tommy, L. Andres, and V. Ricard, "Fifth-generation technologies for the connected car: Capable systems for vehicle-toanything communications," IEEE Veh. Technol. Mag., vol. 13, no. 3, pp. 28-38, Sept. 2018.

[44] M. G. Doone, S. L. Cotton, D. W. Matolak, C. Oestges, S. F. Heaney, and W. G. Scanlon, "Pedestrian-to-vehicle communications in an urban environment: Channel measurements and modeling," IEEE Trans. Antennas Propag., vol. 67, no. 3, pp. 1790-1803, Mar. 2019. 